ESAIM: M2AN 47 (2013) 507-538

DOI: $10.1051 / \mathrm{m} 2 \mathrm{an} / 2012036$
ESAIM: Mathematical Modelling and Numerical Analysis

www.esaim-m2an.org

\title{
AIR ENTRAINMENT IN TRANSIENT FLOWS IN CLOSED WATER PIPES: A TWO-LAYER APPROACH
}

\author{
C. Bourdarias ${ }^{1}$, M. Ersoy $^{2,3}$ and Stéphane Gerbi ${ }^{1}$
}

\begin{abstract}
In this paper, we first construct a model for free surface flows that takes into account the air entrainment by a system of four partial differential equations. We derive it by taking averaged values of gas and fluid velocities on the cross surface flow in the Euler equations (incompressible for the fluid and compressible for the gas). The obtained system is conditionally hyperbolic. Then, we propose a mathematical kinetic interpretation of this system to finally construct a two-layer kinetic scheme in which a special treatment for the "missing" boundary condition is performed. Several numerical tests on closed water pipes are performed and the impact of the loss of hyperbolicity is discussed and illustrated. Finally, we make a numerical study of the order of the kinetic method in the case where the system is mainly non hyperbolic. This provides a useful stability result when the spatial mesh size goes to zero.
\end{abstract}

Mathematics Subject Classification. 74S10, 35L60, 74G15.

Revised May 29, 2012

Published online January 11, 2013.

\section{Notations concerning geometrical quantities}

$\theta(x)$

$Z(x)$

$\Omega_{w}(t, x)$

$\Omega_{a}(t, x)$

$\Omega(x)$

$S(x)$

$R(x)$

$\sigma(x, z)$ angle of the inclination of the main pipe axis $z=Z(x)$ at position $x$

slope

cross-section area of the pipe orthogonal to the axis $z=Z(x)$ filled with water cross-section area of the pipe orthogonal to the axis $z=Z(x)$ filled with air $\Omega(x)=\Omega_{w}(t, x) \cup \Omega_{a}(t, x)$

area of $\Omega(x)$

radius of the cross-section $\Omega(x)$

$\sigma(x, z)=\beta_{r}(x, z)-\beta_{l}(x, z)$ width of the cross-section $\Omega(x)$ at altitude $z$ with $\beta_{r}(x, z)$ (resp. $\beta l(x, z))$ is the right (resp. left) boundary point at altitude $z$.

\footnotetext{
Keywords and phrases. Two-layer vertically averaged flow, free surface water flows, loss of hyperbolicity, nonconservative product, two-layer kinetic scheme, real boundary conditions.

1 Laboratoire de Mathématiques, UMR 5127 - CNRS and Université de Savoie, 73376 Le Bourget-du-Lac Cedex, France. Christian.Bourdarias@univ-savoie.fr; Stephane.Gerbi@univ-savoie.fr

2 BCAM-Basque Center for Applied Mathematics, Bizkaia Technology Park 500, 48160 Derio, Basque Country, Spain.

3 Present address: IMATH-Institut de Mathématiques de Toulon et du Var, Université du sud Toulon-Var, Bâtiment U, BP 20132 - 83957 La Garde Cedex, France. Mehmet.Ersoy@univ-tln.fr
} 


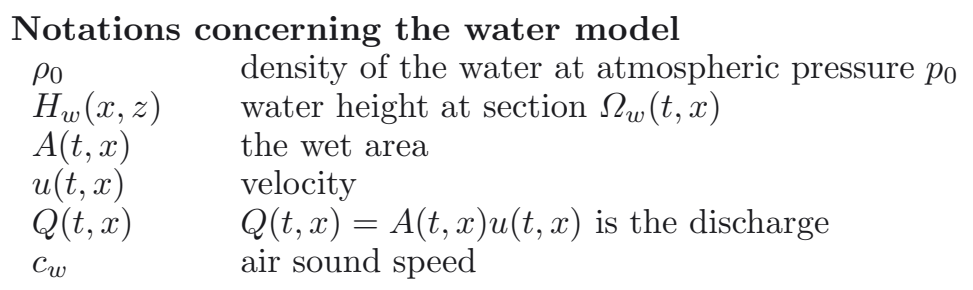

\section{Notations concerning the air model}

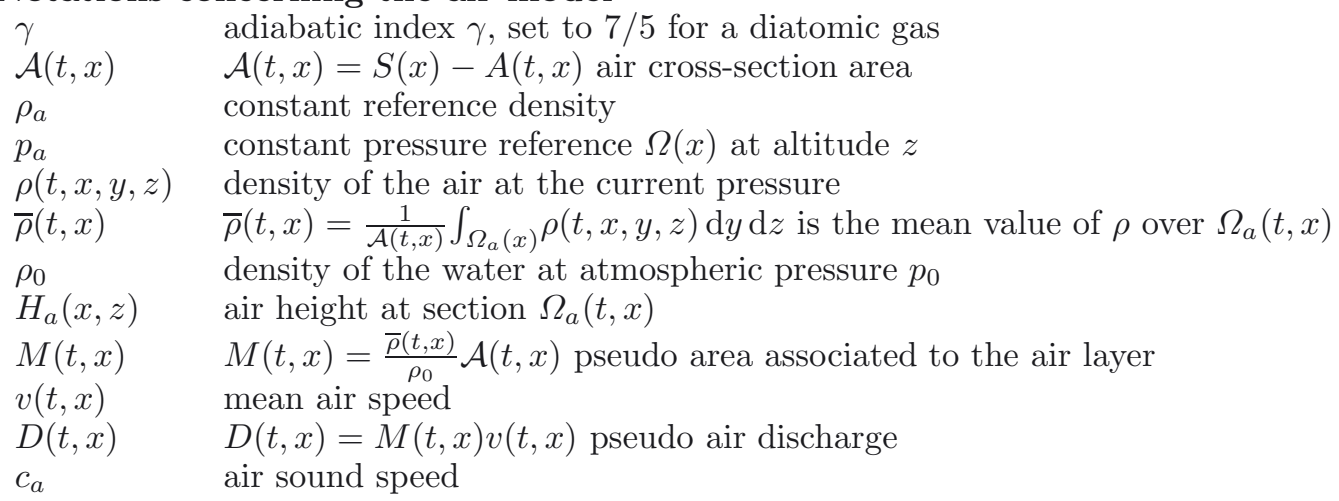

Bold characters are used for vectors and $\alpha$ for both layers when no ambiguities are possible, otherwise $\alpha=a$ for the air layer or $\alpha=w$ for the water layer.

\section{INTRODUCTION}

The hydraulic transients of flow in pipes have been investigated by Hamam and McCorquodale [21], Song et al. [32-34], Wylie and Streeter [38] and others. However, the air flow in a pipeline and the associated air pressure surge was not studied extensively. There is a large literature in which several mathematical models and numerical schemes are presented. Difficulties such as the ill-posedness, the presence of discontinuous fluxes and also the loss of hyperbolicity are studied. This framework enters also in the class of the two-layer shallow water equations. Let us make of a short review of the models that take into account the air and the problem encountered.

The two component gas-liquid mixture flows occur in piping systems in several industrial areas such as nuclear power plants, petroleum industries, geothermal power plants, pumping stations and sewage pipelines. Gas may be entrained in other liquid-carrying pipelines due to cavitation, [37] or gas release from solution due to a drop or increase in pressure.

Unlike in a pure liquid in which the pressure wave velocity is constant, the wave velocity in a gas-liquid mixture varies with the pressure. Thus the main coefficients in the conservation equation of the momentum are pressure dependent and consequently the analysis of transients in the two-component flows is more complex.

The most widely used analytical models for two-component fluid transients are the homogeneous model [14], the drift flux model $[18,22,23]$, and the separated flow model $[13,36]$.

In the homogeneous model, the two phases are treated as a single pseudo-fluid with averaged properties [14,38]: there is no relative motion or slip between the two phases. The governing equations are the same as the one for a single phase flow. However the inertial and gravitational effects can play a more important role than the relative velocity between the air phase and the liquid one: the interaction between the two phases has to be taken into account.

In the drift-flux model, $[18,22,23]$, the velocity fields are expressed in terms of the mixture center-of-mass velocity and the drift velocity of the air phase, which is the air velocity with respect to the volume center of the mixture. The effects of thermal non-equilibrium are accommodated in the drift-flux model by a constitutive 
equation for phase change that specifies the rate of mass transfer per unit volume. Since the rates of mass and momentum transfer at the interfaces depend on the structure of two-phase flows, these constitutive equations for the drift velocity and the vapor generation are functions of flow regimes.

Two-phase flows always involve some relative motion of one phase with respect to the other; therefore, a two-phase flow problem should be formulated in terms of two velocity fields. A general transient two-phase flow problem can be formulated by using a two-fluid model. The separated flow model considers the two phases individually, interacting with each other. Generally, this model will be written as a system of six partial differential equations that represent the balance of mass, momentum and energy $[13,36]$.

A two-phase fluid flow composed of liquid droplets, assumed incompressible, suspended in a compressible gas are governed by an Euler system where each phase are seen as a fluid described by macroscopic quantities is considered in [27]. In this framework, the mass density of the liquid is very large in comparison with the gas mass and have application for cryogenic purposes. The Euler system of six equations, written in a nonconservative form, is hyperbolic for some region depending on the volume fraction, the speed of each phases and the gas sound speed. Sainsaulieu [28] introduces also a finite volume approximation based on an approximate Roe-type Riemann solver which provides good results with the exact solution in one dimension slab geometry for a two phase fluid flows model conditionally hyperbolic.

In [35], the link between two-phase flows and flows in pipes has been studied and the resulting hydrodynamic equations may also possess complex eigenvalues. As a consequence the initial-value problems based on such equations are ill-posed and any consistent finite difference numerical scheme for these equations is unconditionally unstable, i.e., for any constant ratio $\Delta t / \Delta x$, geometrically growing instabilities will always appear if $\Delta x$ is sufficiently small.

Difficulties encountered in this paper enter also in the framework of the so-called two-layer shallow water equations. While nonconservative term implies the lack of appropriate Rankine-Hugoniot relation, the conditionally hyperbolic condition may introduce complex eigenvalues as in the two-phase flows. Moreover, the system is only hyperbolic for small relative speed of each layers for the mode called external wave motion while the internal is specific to these complex eigenvalues. As pointed out by several authors, the violation of such condition is linked to well-known Kelvin-Helmholtz instability, i.e. corresponding to the strong velocity shear between layers, for which the vertically averaged two-layer system is not a priori suitable (see for instance [1]).

Several attempts have been proposed to solve these problems. We may cite the numerical scheme proposed by Abgrall and Karni [1] which deals with nonconservative and non hyperbolic system by using relaxation approach which offers a greater decoupling and provides an access to the eigenstructure. We refer to [25] for discussion of numerical schemes and to [4] for the recent progress on hyperbolic systems with source terms.

Finally, although questions about the ill-posedness equations remain unsettled, such a priori ill-posed equations are widely used in practice such as for multiphase-flow computations, backward heat conduction and porous media flows.

In the present paper, we consider the air entrainment appearing in the transient flow in closed pipes filled by the free surface flow and air flow. We derived the present model in a very closed manner as the derivation of SaintVenant equations for free surface open channel flows: we consider that the liquid is inviscid and incompressible (thus Euler equations are used) and its pressure is only due to the gravitational effect. It is the well known hydrostatic pressure law. A kinematic law at the free surface and a no-leak condition are used to complete the Euler system. The air phase is supposed to be compressible and isothermal: thus we can use an equation of state of the form $p=k \rho^{\gamma}$ where $\gamma$ represents the adiabatic exponent. To connect the two phases, we write the continuity of the normal stress tensor at the free surface separating the two phases: the hydrostatic pressure law for the fluid is thus coupled to the pressure law governing the air flow at the free surface. Then by taking averaged values over cross-sections of the main flow axis, we obtain a system of four partial differential equations which is conditionally hyperbolic: we call it the two-layer water/air model. In Theorem 2.3, we state its main property: the existence of a total energy. The derivation of the proposed model and the analysis of the mathematical properties are presented in Section 2 as well as a careful study of the eigenvalues of the air-layer model: the 
problem is hyperbolic for small relative speed but also for large relative speed which was already pointed out in the pioneer work of [24] and recalled later on by Barros and Choi [3] and the reference therein.

As in $[9,11,26]$, we choose to interpret this model as a kinetic model since the computation of eigenvalues are not required: we present the mathematical kinetic formulation of the system of four partial differential equations in Section 3.

This mathematical formulation allows us to introduce the two-layer kinetic scheme presented in Section 4 which allows to reproduce fairly physical solutions even if the system loses its hyperbolicity. To deal with real boundary conditions, we also introduce in Section 4.2 the "decoupled boundary conditions" based on the solution of the Riemann problem for the decoupled system for which the eigenstructure is completely well-defined.

Finally, we propose in Section 5 some numerical simulations to focus on the role of the air entrainment on the free surface fluid flow and the behavior of the numerical solutions provided for internal wave motion, i.e. when the system is not hyperbolic. More precisely, even if the presented two-layer system is not hyperbolic, the numerical scheme seems to be stable and there are no geometric instabilities for a large series of numerical tests. This surprising stability is certainly due to the fact that the energy is conserved as stated in Theorem 2.3. The scheme is then stable with respect to smaller $\Delta x$ and produces, even is the decay rate is worse than the case where the air is not taken into account, usable numerical results.

\section{A TWO-LAYER WATER/AIR MODEL}

We will consider throughout this paper a transient flow in a closed pipe that is not completely filled of water nor of air. The flow is composed by two air and water layers separated by a moving interface. We assume that the two layers are immiscible. We will consider that the fluid is perfect and incompressible whereas the air is a perfect compressible gas: we neglect here the thermodynamic relations such as the air-water interactions as condensation/evaporation since we suppose that the air layer follows an isothermal process and is assumed to be isentropic.

The derivation of the two-layer model is then performed with the use of the 3D Euler (incompressible for the fluid, compressible for the gas) equations where the no-leak condition is assumed for the fluid as well as for the gas at the pipe boundary. The continuity of the pressure at the interface separating each layers is assumed.

Remark 2.1. Throughout the paper, we use the following notations: we will denote by the index $w$ the water layer and by the index $a$ the air layer. We also use the index $\alpha$ to write indifferently the liquid or gas layer.

The pipe is assumed to have a symmetry axis $\mathcal{C}$, says circular one, which is represented by a straight line with a constant angle $\theta .(O, \mathbf{i}, \mathbf{j}, \mathbf{k})$ is a reference frame attached to this axis with $\mathbf{k}$ orthogonal to $\mathbf{i}$ in the vertical plane containing $\mathcal{C}$, see Figure 1a. We denote by $\Omega_{t, a}$ and $\Omega_{t, w}$ the gas and fluid domain occupied at time $t$ as represented on Figure 1. Then, at each point $\omega(x)$, we define the water section $\Omega_{w}(t, x)$ by:

$$
\Omega_{w}(t, x)=\left\{(y, z) \in \mathbb{R}^{2} ; z \in\left[-R(x),-R(x)+H_{w}(t, x)\right], y \in\left[\beta_{l}(x, z), \beta_{r}(x, z)\right]\right\}
$$

and the air one by:

$$
\Omega_{a}(t, x)=\left\{(y, z) \in \mathbb{R}^{2} ; z \in\left[-R(x)+H_{w}(t, x), R(x)\right], y \in\left[\beta_{l}(x, z), \beta_{r}(x, z)\right]\right\}
$$

where $R(x)$ stands for the radius, $H_{w}(t, x)$ the water height at section $\Omega_{w}(t, x), H_{a}(t, x)$ the air height at section $\Omega_{a}(t, x) . \beta_{l}(x, z), \beta_{r}(x, z)$ are respectively the left and right $y$ values of the boundary points of the domain at altitude $-R(x)<z<R(x)$ (see Figs. 1a and 1b).

We have then the first natural coupling:

$$
H_{w}(t, x)+H_{a}(t, x)=2 R(x) .
$$

In what follows, we will denote $h_{w}(t, x)=-R(x)+H_{w}(t, x)$ the algebraic water height. 


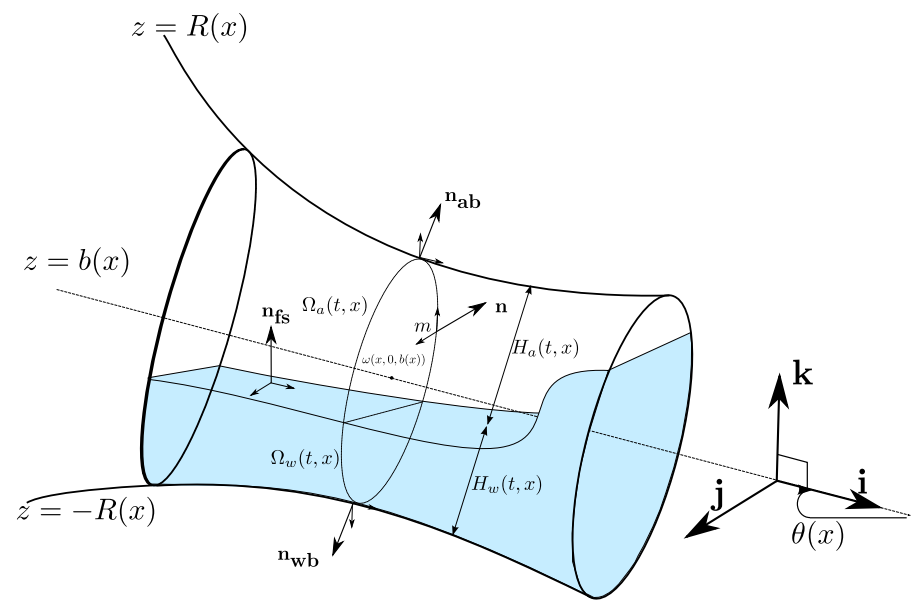

(a) Geometric characteristics of the domain

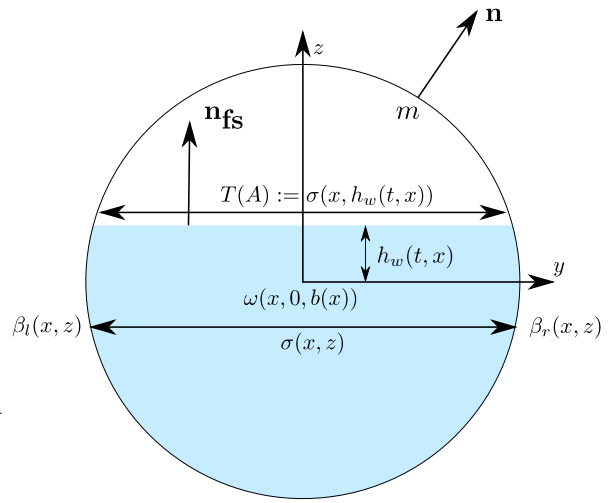

(b) Cross-section of the domain

FiguRE 1. Geometry of the pipe: notations and settings.

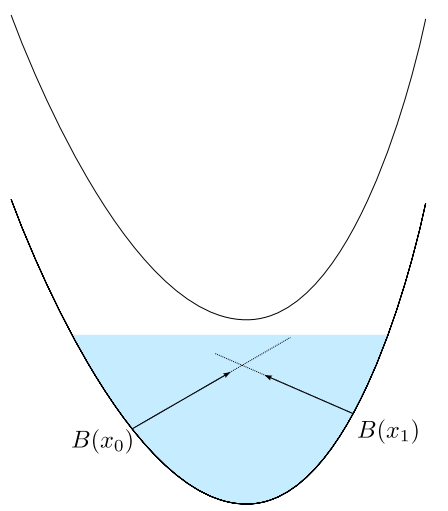

FiguRE 2. Geometric restriction on the pipe for curved ground.

Remark 2.2. Let us emphasize that the assumption $\theta=$ cst is made for the sake of simplicity since the variable case does not introduce novelty with respect to the reference therein. Nevertheless, let us note that the curved ground case (see, for instance, [10,29] and [17], Chap. 1) is adapted by introducing the assumption on the curvature radius $\mathcal{R}(x)$, i.e. $\frac{\mathcal{R}(x)}{R(x)}>1$ where $R(x)$ is the radius of the pipe at $x$ along the curvilinear frame. This hypothesis ensure that the transformation of the initial Euler equations written in a given frame to the curvilinear one is a diffeomorphism and avoid the situation as illustrated in Figure 2. We refer to [10] for a detailed derivation of it.

\subsection{The fluid free surface model}

The shallow water equations for free surface flows are obtained from the incompressible Euler equations (2.2) which writes in cartesian coordinates $(x, y, z)$ :

$$
\begin{aligned}
& \operatorname{div}\left(\mathbf{U}_{\mathbf{w}}\right)=0, \quad \text { on } \mathbb{R} \times \Omega_{t, w} \\
& \partial_{t}\left(\mathbf{U}_{\mathbf{w}}\right)+\operatorname{div}\left(\mathbf{U}_{\mathbf{w}} \otimes \mathbf{U}_{\mathbf{w}}\right)+\nabla P_{w}=\mathbf{F}, \quad \text { on } \mathbb{R} \times \Omega_{t, w}
\end{aligned}
$$


where $\mathbf{U}_{\mathbf{w}}(t, x, y, z)=\left(U_{w}, V_{w}, W_{w}\right), P_{w}(t, x, y, z), \mathbf{F}$ stand for, respectively, the fluid velocity, the scalar pressure and the exterior strength of gravity.

A kinematic law at the free surface and a no-leak condition $\mathbf{U}_{\mathbf{w}} \cdot \mathbf{n}_{\mathbf{w b}}=0$ on the wet boundary complete the system $\left(\mathbf{n}_{\mathbf{w b}}\right.$ being the outward unit normal vector, see Fig. $\left.1 b\right)$.

To model the effects of the air on the water, we write the continuity of the normal stress at the boundary layer separating the two phases. Thus, the water pressure $P_{w}$ may be written as:

$$
P_{w}=g\left(h_{w}-z\right) \cos \theta+\frac{P_{a}}{\rho_{0}}
$$

where the term $g\left(h_{w}-z\right) \cos \theta$ is the hydrostatic pressure, $\rho_{0}$ is the density of water at atmospheric pressure, $P_{a}$ is the air pressure. This is the second natural coupling between layers.

The Euler equations (2.2) will be reduced to a shallow water like equations using standard arguments. Let us briefly recall for the convenience of the reader the setup of the derivation (see [10,17], Chap. 1, for the detailed derivation).

The aspect-ratio defined by

$$
\varepsilon=\frac{2 R}{L} \ll 1
$$

is assumed small where $L$ and $R$ stands for the characteristic length and the radius of the pipe. Following the classical thin-layer asymptotic analysis of Gerbeau and Perthame [20] and [10,17], Chapter 1, we introduce $\bar{U}$, $\bar{V}, \bar{W}$ respectively, the characteristic horizontal, normal and binormal speed with respect to the moving frame such that $\epsilon=\frac{\bar{V}}{\bar{U}}=\frac{\bar{W}}{\bar{U}}, \bar{U}=\frac{L}{T}, P=\bar{U}^{2}$ for some characteristic time $T$.

We also introduce the non dimensional variables $\frac{U}{U}, \varepsilon \frac{V}{\bar{U}}, \varepsilon \frac{W}{\bar{U}}$, being respectively, the horizontal, normal and binormal non dimensional speed with respect to the moving frame with $\frac{x}{L}, \frac{y}{H}, \frac{z}{H}, \frac{p}{P}, \frac{\theta}{\theta_{0}}$ respectively, the non dimensional $x, y, z$ coordinate, the non dimensional pressure and the non dimensional angle.

Rescaling the Euler equations (2.2) with these non dimensional variables, one can formally take $\varepsilon=0$ to get the hydrostatic approximation. Then, we introduce the conservative variables $A(t, x)$ and $Q(t, x)=A(t, x) u(t, x)$ representing respectively the wet area and the discharge defined as:

$$
A(t, x)=\int_{\Omega_{w}} \mathrm{~d} y \mathrm{~d} z, \quad Q(t, x)=A(t, x) u(t, x)
$$

where $u$ is the mean value of the speed

$$
u(t, x)=\frac{1}{A(t, x)} \int_{\Omega_{w}} U_{w}(t, x, y, z) \mathrm{d} y \mathrm{~d} z .
$$

As done by the authors in [10], taking the averaged values along the cross-section $\Omega_{w}(t, x)$ in the hydrostatic approximation of the incompressible Euler equations, and approximating the averaged value of a product by the product of the averaged values, yields to the free surface model:

$$
\left\{\begin{aligned}
\partial_{t} A+\partial_{x} Q & =0 \\
\partial_{t} Q+\partial_{x}\left(\frac{Q^{2}}{A}+A P_{a} / \rho_{0}+g I_{1}(x, A) \cos \theta\right) & =-g A \partial_{x} Z+g I_{2}(x, A) \cos \theta+P_{a} / \rho_{0} \partial_{x} A .
\end{aligned}\right.
$$

where $g$ is the gravity constant and $Z(x)$ is the elevation of the point $\omega(x)$. The terms $I_{1}(x, A)$ and $I_{2}(x, A)$ are defined by:

$$
I_{1}(x, A)=\int_{-R}^{h_{w}}\left(h_{w}-Z\right) \sigma(x, z) \mathrm{d} z
$$


and

$$
I_{2}(x, A)=\int_{-R}^{h_{w}}\left(h_{w}-Z\right) \partial_{x} \sigma(x, z) \mathrm{d} z .
$$

They represent respectively the term of hydrostatic pressure and the pressure source term. In theses formulas $\sigma(x, z)$ is the width of the cross-section at position $x$ and at height $z$ (see Fig. 1b).

Remark 2.3. In view of Remark 2.2, whenever $\theta=\theta(x)$, an additional curvature source term of the form $\bar{z}(\cos \theta)^{\prime}$ appears where $\bar{z}=h_{w}(A)-\frac{I_{1}(x, A)}{A}$ stands for the distance separating the free surface to the center of the mass of the fluid (see [10,17], Chap. 1).

\subsection{The air layer model}

The derivation of the air layer model is based on the Euler compressible equations (2.5) which writes in Cartesian coordinates $(x, y, z)$ :

$$
\begin{aligned}
\partial_{t} \rho+\operatorname{div}\left(\rho \mathbf{U}_{\mathbf{a}}\right)=0, & \text { on } \mathbb{R} \times \Omega_{t, a} \\
\partial_{t}\left(\rho \mathbf{U}_{\mathbf{a}}\right)+\operatorname{div}\left(\rho \mathbf{U}_{\mathbf{a}} \otimes \mathbf{U}_{\mathbf{a}}\right)+\nabla P_{a}=0, & \text { on } \mathbb{R} \times \Omega_{t, a}
\end{aligned}
$$

where $\mathbf{U}_{\mathbf{a}}(t, x, y, z)=\left(U_{a}, V_{a}, W_{a}\right)$ and $\rho(t, x, y, z)$ denotes the velocity and the density of the air whereas $P_{a}(t, x, y, z)$ is the scalar pressure. Let us mention here that we neglect the gravitational effect on the air layer.

We assume that the air layer is isentropic, isothermal and follows a perfect gas law. Thus we have the following equation of state:

$$
P_{a}(\rho)=k_{a} \rho^{\gamma} \text { with } k_{a}=\frac{p_{a}}{\rho_{a}^{\gamma}}
$$

for some reference pressure $p_{a}$ and density $\rho_{a}$. The adiabatic index $\gamma$ is set to $7 / 5$ for a diatomic gas and the constant $k_{a}$ depends on the universal constant $R_{u c}$ and the temperature.

Remark 2.4. The definition of the free surface pressure (2.3) and the air pressure (2.6) ensures the continuity property of the normal stress for two immiscible perfect fluids at the interface.

Taking advantage of the geometric configuration as in the previous subsection, i.e. the aspect-ratio $\varepsilon=2 R / L$ being small, we proceed to a thin-layer asymptotic approximation following the derivation of the pressurized model (used in the context of the unsteady mixed flows in closed water pipes) [10,17], Chapter 1, which has the same structure as the Euler equations (2.5) used here. Indeed, only the index $\gamma$ changes from one model to this one.

The thin-layer asymptotic settings are essentially the same as used in the previous subsection. Thus, in the following development, we assume that the density of air and the pressure depends only on $t$ and $x$, i.e. we use this notation when no ambiguity occurs.

Let us now introduce the air area $\mathcal{A}$ by:

$$
\mathcal{A}=\int_{\Omega_{a}} \mathrm{~d} y \mathrm{~d} z
$$

the averaged air velocity $v$ (we recall that $u$ is the averaged velocity of the fluid layer) by:

$$
v(t, x)=\frac{1}{\mathcal{A}(t, x)} \int_{\Omega_{a}} U_{a}(t, x, y, z) \mathrm{d} y \mathrm{~d} z,
$$

and the averaged density by:

$$
\bar{\rho}(t, x)=\frac{1}{\mathcal{A}(t, x)} \int_{\Omega_{a}} \rho(t, x, y, z) \mathrm{d} y \mathrm{~d} z .
$$

The conservative variables are $M=\bar{\rho} / \rho_{0} \mathcal{A}$ ("pseudo area occupied by the air") and $D=M v$ ("pseudo air discharge"). Let us remark that we use rescaled mass and discharge instead of the real mass and discharge to be 
consistent with the free surface model (2.4). As done before to obtain the water layer model, we take averaged values in the Euler equations (2.5) over sections $\Omega_{a}(t, x)$, and perform the same approximations on averaged values of a product to get the following model:

$$
\left\{\begin{aligned}
\partial_{t} M+\partial_{x} D & =\int_{\partial \Omega_{a}} \rho / \rho_{0}\left(\partial_{t} \mathbf{m}+v \partial_{x} \mathbf{m}-\mathbf{v}\right) \cdot \mathbf{n} \mathrm{d} s \\
\partial_{t} D+\partial_{x}\left(\frac{D^{2}}{M}+P_{a}(\bar{\rho}) / \rho_{0} \mathcal{A}\right) & =P_{a}(\bar{\rho}) / \rho_{0} \partial_{x}(\mathcal{A})+\int_{\partial \Omega_{a}} \rho / \rho_{0} v\left(\partial_{t} \mathbf{m}+v \partial_{x} \mathbf{m}-\mathbf{v}\right) . \mathbf{n} \mathrm{d} s
\end{aligned}\right.
$$

where $\mathbf{v}$ is the velocity in the $(\mathbf{j}, \mathbf{k})$-plane. The boundary $\partial \Omega_{a}$ is divided into the free surface boundary $\Gamma_{f s}$ and the border of the pipe in contact with air $\Gamma_{c}$. For $m \in \Gamma_{c}, \mathbf{n}=\frac{\mathbf{m}}{|\mathbf{m}|}$ is the outward unit vector at the point $m$ in the $\Omega$-plane and $\mathbf{m}$ stands for the vector $\omega m$ while $\mathbf{n}$ denotes $-\mathbf{n}_{f s}$ on $\Gamma_{f s}$. As the pipe is infinitely rigid, the non-penetration condition holds on $\Gamma_{c}$, namely: $\mathbf{U}_{\mathbf{a}} \cdot \mathbf{n}_{a b}=0$ (see Fig. 1a).

On the free surface, as the boundary kinematic condition used for the water layer at the free surface holds, the integral appearing in system (2.7) vanishes. Using the equation of state (2.6), and using the air sound speed defined by:

$$
c_{a}^{2}=\frac{\partial p}{\partial \rho}=k_{a} \gamma\left(\frac{\rho_{0} M}{\mathcal{A}}\right)^{\gamma-1},
$$

we finally get the air-layer model:

$$
\left\{\begin{aligned}
\partial_{t} M+\partial_{x} D & =0 \\
\partial_{t} D+\partial_{x}\left(\frac{D^{2}}{M}+c_{a}^{2} \frac{M}{\gamma}\right) & =c_{a}^{2} \frac{M}{\gamma} \frac{\partial_{x}(\mathcal{A})}{\mathcal{A}} .
\end{aligned}\right.
$$

\subsection{The two-layer model}

The two-layer averaged flow model is then simply obtain by the apposition of the models (2.4) and (2.9), using the coupling (2.1) which also writes $\mathcal{A}+A=S$ where $S=S(x)$ denotes the pipe section and using the definition of the air pressure (2.6) and the air sound speed (2.8), we get:

$$
\left\{\begin{aligned}
\partial_{t} M+\partial_{x} D & =0, \\
\partial_{t} D+\partial_{x}\left(\frac{D^{2}}{M}+c_{a}^{2} \frac{M}{\gamma}\right) & =c_{a}^{2} \frac{M}{\gamma} \frac{\partial_{x}(S-A)}{(S-A)}, \\
\partial_{t} A+\partial_{x} Q & =0, \\
\partial_{t} Q+\partial_{x}\left(\frac{Q^{2}}{A}+g I_{1}(x, A) \cos \theta+A \frac{c_{a}^{2} M}{\gamma(S-A)}\right) & =-g A \partial_{x} Z+g I_{2}(x, A) \cos \theta \\
& +\frac{c_{a}^{2} M}{\gamma(S-A)} \partial_{x} A .
\end{aligned}\right.
$$

\subsection{Study of the eigenvalues of the two-layer model}

As for almost every two-layer shallow water models $[1,5,12,30]$ as well as two phase fluid flows $[22,23,27,32,35]$, our model is a conditionally hyperbolic system as we will see thereafter. By definition, it means that with respect to some physical parameters, for instance, when the relative speed between the two layers is small the system remains hyperbolic as already pointed out by several authors (see for instance [1,5,27]). In fact, this property is not only restricted to small relative speed but holds also for large one as it was emphasized by Ovsjannikov [24] and recalled later on by Barros and Choi [3] and the reference therein.

In the present case, the coupling between the layers, due to the hydrostatic pressure (2.3) and the barotropic one (2.6), provides a full access to the the computation of the eigenvalues, which is required for almost every numerical schemes based on Riemann solver. 
Noting $\mathbf{W}=(M, D, A, Q)^{t}$ the unknown vector, the system may be written in the quasilinear form:

$$
\partial_{t} \mathbf{W}+\mathcal{D}(x, \mathbf{W}) \partial_{X} \mathbf{W}=0
$$

with the convection matrix:

$$
\mathcal{D}=\left(\begin{array}{cccc}
0 & 1 & 0 & 0 \\
c_{a}^{2}-v^{2} & 2 v & \frac{M}{S-A} c_{a}^{2} & 0 \\
0 & 0 & 0 & 1 \\
\frac{A}{(S-A)} c_{a}^{2} & 0 & c_{w}^{2}+\frac{A M}{(S-A)^{2}} c_{a}^{2}-u^{2} 2 u
\end{array}\right)
$$

contains flux gradient terms for which the quantities $c_{a}$ stands for the air sound speed defined by (2.8) and $c_{w}=\sqrt{g_{\frac{A}{T(A)} \cos \theta}}$ the water sound speed where $T(A)$ is the width of the free surface at height $h_{w}(A)$ (see Fig. 1b).

Remark 2.5. Let us emphasize that $c_{w}$ stands for the classical water sound speed when the air layer is not taken into account. The new quantity $c_{m}^{2}:=c_{w}^{2}+\frac{A M}{(S-A)^{2}} c_{a}^{2}$ represents the square of the water sound speed which takes into account the air effect.

The characteristic polynomial of degree 4 of the convection matrix $\mathcal{D}$ of the two-layer system is defined by:

$$
P(\lambda)=\left(\lambda^{2}-2 v \lambda-\left(c_{a}^{2}-v^{2}\right)\right)\left(\lambda^{2}-2 u \lambda-\left(c_{w}^{2}+\frac{A M}{(S-A)^{2}} c_{a}^{2}-u^{2}\right)\right)-\frac{A M}{(S-A)^{2}} c_{a}^{4}
$$

for which no simple expression for the eigenvalues can be computed but one can provide, as it was already done in the context of the two-layer shallow water model, the first order expansion with respect to the relative speed between layers (see for instance $[2,31]$ ) and derive an hyperbolicity condition which may also linked to the Kelvin-Helmholtz instability.

In what follows, we define the following non dimensional numbers:

$$
F=\frac{v-u}{c_{m}}, \quad \sqrt{H}=\frac{c_{a}}{c_{m}}, \quad c_{m}=\sqrt{c_{w}^{2}+s c_{a}^{2}} \quad \text { with } \quad s=\frac{A M}{(S-A)^{2}} \geqslant 0 .
$$

$F$ stands for the relative speed between the two layers, $H$ represents the ratio between the sound speed of each layer and $s=\frac{\rho}{\rho_{0}} \frac{A}{S-A}$ represents the compressibility ratio between the air layer and the water layer.

In Section 3.1, based on the kinetic formulation of the two-layer model (2.10), we introduce a new numerical scheme which does not require the computation of the eigenvalues and therefore we will focus on its stability and sensitivity when the system is non hyperbolic. This motivates the following study.

Remark 2.6. Let us firstly remark that although, for the construction of the kinetic scheme we are not interested in the the computation of these eigenvalues, one can provide easily the expression of them when the relative speed is equal to zero i.e. $F=0$ :

$$
u \pm \frac{1}{2} \sqrt{2 c_{a}^{2}+2 c_{m}^{2} \pm \sqrt{\left(c_{a}^{2}-c_{m}^{2}\right)^{2}+4 s c_{a}^{4}}}
$$

for which we immediately see that, we may have complex eigenvalues as observed in Figures $4 \mathrm{a}$ and $4 \mathrm{~b}$. When $H \geq 1 / 4 s$ eigenvalues are real, otherwise two eigenvalues are real and two are complex. Moreover, in comparison with the two-layer shallow water for instance, the condition "relatively small enough" is not sufficient to state that the present system is hyperbolic. This is due to the fact that $s$ is not bounded from above (as we will see).

Let us recall the so-called root location criteria for a polynomial of degree 4 (see also [19]). 
Theorem 2.1. Let $P$ be a polynomial of degree 4 defined by:

$$
P(x)=\sum_{k=0}^{4} a_{k} x^{4-k} \quad \text { for } \quad\left(a_{k}\right)_{k} \in \mathbb{R} \quad \text { and } \quad a_{0}>0 .
$$

All the root of equation (2.13) are real if and only if one of the following conditions holds:

(1) $\Delta_{3}>0, \Delta_{5}>0$ and $\Delta_{7} \geqslant 0$;

(2) $\Delta_{3} \geqslant 0, \Delta_{5}=0$ and $\Delta_{7}=0$.

Where $\Delta_{3}, \Delta_{5}, \Delta_{7}$ are the determinant of the matrices:

$$
\Delta_{3}=\left(\begin{array}{ccc}
a_{0} & a_{1} & a_{2} \\
0 & 4 a_{0} & 3 a_{1} \\
4 a_{0} & 3 a_{1} & 2 a_{2}
\end{array}\right), \quad \Delta_{5}=\left(\begin{array}{ccccc}
a_{0} & a_{1} & a_{2} & a_{3} & a_{4} \\
0 & a_{0} & a_{1} & a_{2} & a_{3} \\
0 & 0 & 4 a_{0} & 3 a_{1} & 2 a_{2} \\
0 & 4 a_{0} & 3 a_{1} & 2 a_{2} & a_{3} \\
4 a_{0} & 3 a_{1} & 2 a_{2} & a_{3} & 0
\end{array}\right)
$$

and

$$
\Delta_{7}=\left(\begin{array}{ccccccc}
a_{0} & a_{1} & a_{2} & a_{3} & a_{4} & 0 & 0 \\
0 & a_{0} & a_{1} & a_{2} & a_{3} & a_{4} & 0 \\
0 & 0 & a_{0} & a_{1} & a_{2} & a_{3} & a_{4} \\
0 & 0 & 0 & 4 a_{0} & 3 a_{1} & 2 a_{2} & a_{3} \\
0 & 0 & 4 a_{0} & 3 a_{1} & 2 a_{2} & a_{3} & 0 \\
0 & 4 a_{0} & 3 a_{1} & 2 a_{2} & a_{3} & 0 & 0 \\
4 a_{0} & 3 a_{1} & 2 a_{2} & a_{3} & 0 & 0 & 0
\end{array}\right)
$$

Let us now apply this result to the characteristic equation (2.11). To this end, we closely follow the study of Barros and Choi [3]. We thus introduce the non-dimensional variable $x=\lambda / c_{m}$ and as mentioned by Barros and Choi, without loss of generality, we may assume that $u / c_{m}=1$ (by choosing a moving reference frame such that this condition is met). Then equation (2.11) becomes:

$$
P(x)=x^{4}-2(2+F) x^{3}+((1+F)(5+F)-H) x^{2}+2\left(H-(1+F)^{2}\right) x-s H^{2} .
$$

Let us first remark that $\Delta_{3}=4\left(F^{2}+2(1+H)\right)$ is always strictly positive.

Secondly, we have:

$$
\Delta_{5}=8\left((1+H) F^{4}-2\left(H^{2}(1-s)+1-6 H\right) F^{2}+(1+H)\left((H-1)^{2}+4 s H^{2}\right)\right) .
$$

Thus $\Delta_{5}$ is a polynomial of degree 2 in the variable $y=F^{2}$. Its discriminant $R(H, s)$ is equal to:

$$
R(H, s)=256 H\left(\left(s^{2}-6 s\right) H^{3}+(4 s-12) H^{2}+(40-6 s) H-12\right)
$$

which is a polynomial of degree 4 in the variable $H$. Since $8(1+H)$ (which is the dominant coefficient of $\Delta_{5}$ ) is positive, we are firstly interested in the set where the discrimant $R(H, s)$ is negative.

Since $s \geqslant 0, R(H, s)$ admits four real roots:

$$
l_{1}(s)=\frac{-1+\sqrt{1+6 s}}{s}, l_{2}(s)=-\frac{1+\sqrt{1+6 s}}{s}, l_{3}(s)=-\frac{2}{s-6}, \quad \text { and } \quad l_{4}(s)=0 .
$$

Let $\mathcal{D}$ bet the set defined by:

$$
\mathcal{D}=\left\{(H, s) \in \mathbb{R}_{+}^{2} ; \begin{array}{l}
0<s<4 \text { and } l_{3}(s)<H<l_{1}(s) \\
\text { or } 4<s<6 \text { and } l_{1}(s)<H<l_{3}(s),
\end{array}\right\} .
$$




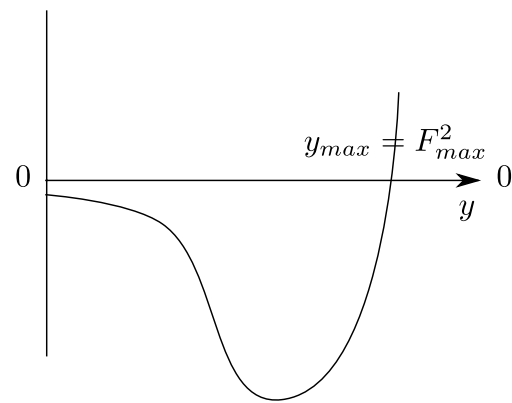

(a) $y_{\min }<0$

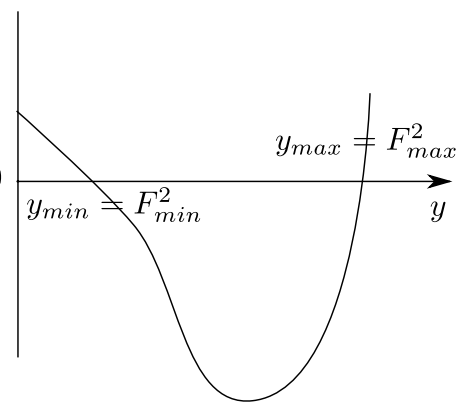

(b) $y_{\min }>0$

FiguRE 3. Behavior of the polynomial $Q(y)$.

If $(H, s) \in \mathcal{D}$, then $R(H, s) \leqslant 0$ and $\Delta_{5}>0$. On the contrary, if $(H, s) \notin \mathcal{D}$ then $R(H, s) \geqslant 0$ and $\Delta_{5}$ admits two real eigenvalues which are both negative:

$$
y_{ \pm}=\frac{2\left(H^{2}(1-s)+1-6 H\right) \pm \sqrt{R(H, s)}}{(1+H)}<0 \quad \text { for } \quad(H, s) \notin \mathcal{D} .
$$

By definition, we have $y=F^{2}$ and thus for every $(H, s) \in \mathbb{R}^{2}, \Delta_{5}>0$. Therefore we must only examine the sign of $\Delta_{7}$.

$\Delta_{7}$ is a polynomial of degree 4 in the variable $y=F^{2}$ and we write it as $\Delta_{7}(y)=16 H Q(y)$ where

$$
\begin{aligned}
Q(y)= & y^{4}+\left(s H^{2}+(s-4) H-4\right) y^{3}+\left(\left(s^{2}-3 s\right) H^{3}+(6-26 s) H^{2}+(4-3 s) H+6\right) y^{2} \\
& +\left(\left(3 s-20 s^{2}\right) H^{4}+\left(13 s-20 s^{2}-4\right) H^{3}+(13 s+4) H^{2}+(4 s+3) H-4\right) y \\
& -\left(16 s^{3}+8 s^{2}+s\right) H^{5}+\left(32 s^{2}+12 s+1\right) H^{4}-\left(4+22 s+8 s^{2}\right) H^{3}+(12 s+6) H^{2}-(4+s) H+1 .
\end{aligned}
$$

We have to study the sign of $\Delta_{7}(y)$ only for $y \geq 0$. Using a symbolic computation software such as Maxima ${ }^{\circledR}$, we obtain:

$$
\Delta_{3}(Q)>0, \Delta_{5}(Q) \leqslant 0 \text { and } \Delta_{7}(Q)>0 .
$$

Applying again Theorem 2.1 (see also Fuller [19], Thms. 2 and 4) for the polynomial $Q$, it exists $y_{\min }(H, s) \in \mathbb{R}$ which may be positive or negative and $y_{\max }(H, s)>0$ (which are the two real roots of $Q$ ) such that:

$$
\forall y \in\left(-\infty, y_{\min }\right] \cup\left[y_{\max }, \infty\right), Q(y) \geq 0 .
$$

We have illustrated this property in Figure 3.

We can now summarized the study of the hyperbolicity of the two-layer model by the following result:

Theorem 2.2. Given a couple $(s, H)$, the two-layer system is hyperbolic whenever $y=F^{2}$ satisfies one of the following conditions:

(1) $y_{\min }(H, s) \leqslant 0$ and $y \geqslant y_{\max }$;

(2) $y_{\min }(H, s)>0$ and $y \in\left[0, y_{\min }\right] \cup\left[y_{\max }, \infty\right)$.

Remark 2.7. Let us conclude this study about the hyperbolicity of the two layer model by the following remarks.

(1) The criterion $y=F^{2} \geqslant F_{\max }^{2}:=y_{\max }$ ensures the hyperbolicity for large relative speed while when $y_{\min }>0$ the condition $0 \leqslant F^{2} \leqslant y_{\min }=F_{\min }^{2}$ corresponds to small relative speed. 


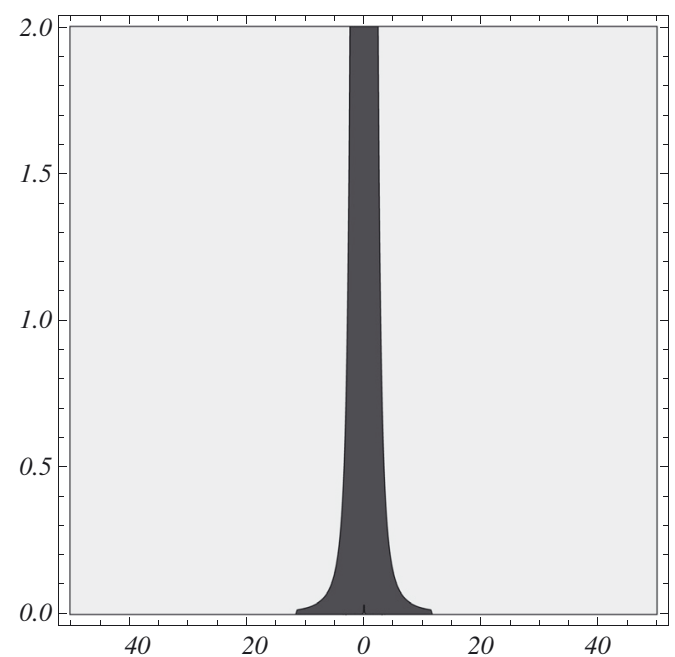

(a) $\rho=1000, F x$-axis and $A y$-axis

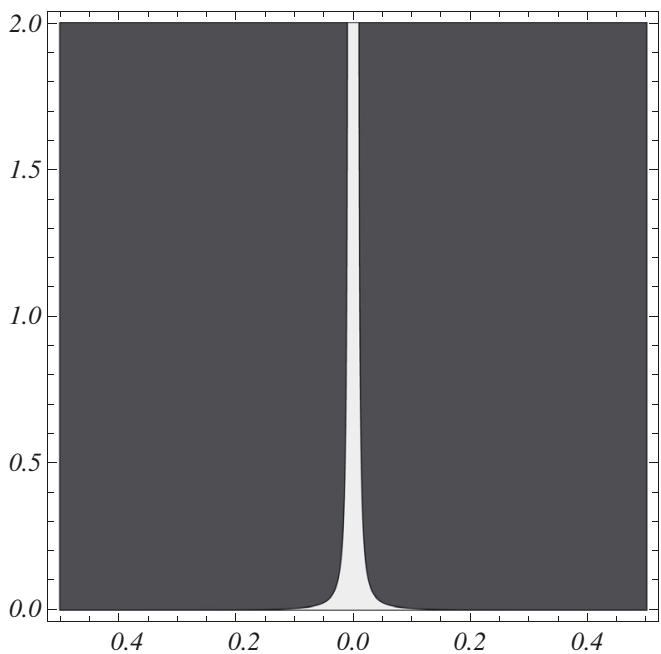

(b) Zoom on : $\rho=1000, F x$-axis and $A y$-axis

FiguRE 4. Small and large relative speed: hyperbolic region.

(2) The term $s=\frac{\rho}{\rho_{0}} \frac{A}{S-A}$ is the equivalent ratio $\frac{\rho_{2}}{\rho_{1}}<1$ appearing in the two-layer shallow water equations, see [3]. Here $s$ is not bounded from above thus $y_{\min }$ is not necessary positive.

(3) Since $s=s(\rho, A)$ and $H=H(\rho, A)$, the preceding result can be formulated in terms of the following three parameters $F, \rho$ and $A$. For different values of these parameters, we may draw the region where one of the preceding conditions is fulfilled.

In Figures 4 and 5 the black gray represents the non hyperbolic region.

In Figures $4 \mathrm{a}$ and $4 \mathrm{~b}$, we represent the hyperbolic region part for small relative speed between the two layers (that is for large value of $\rho$ ) while in Figure 5a we represent the hyperbolic region part for large relative speed between the two layers (that is for small value of $\rho$ ). We see in Figure 5 a that the thickness of the hyperbolic region decreases with $\rho$. Finally we also represent on Figure $5 \mathrm{~b}$ the hyperbolic region versus the three parameters $F, \rho$ and $A$.

(4) We conclude the preceding numerical study of the eigenvalues by noticing that for small and large relative speed, the two layer model remains hyperbolic.

Finally, even if System (2.10) is conditionally hyperbolic, the existence of a convex entropy function is not a contradiction. Consequently, admissible weak solutions should satisfy the corresponding below-mentioned entropy inequality (see for instance $[1,5]$ ).

The results are summarized in the following theorem:

\section{Theorem 2.3.}

(1) For smooth solutions of (2.10), the velocities $u$ and $v$ satisfy

$$
\begin{aligned}
\partial_{t} v+\partial_{x}\left(\frac{v^{2}}{2}+\frac{c_{a}^{2}}{\gamma-1}\right) & =0, \\
\partial_{t} u+\partial_{x}\left(\frac{u^{2}}{2}+g h_{w}(A) \cos \theta+g Z+\frac{c_{a}^{2} M}{\gamma(S-A)}\right) & =0 .
\end{aligned}
$$




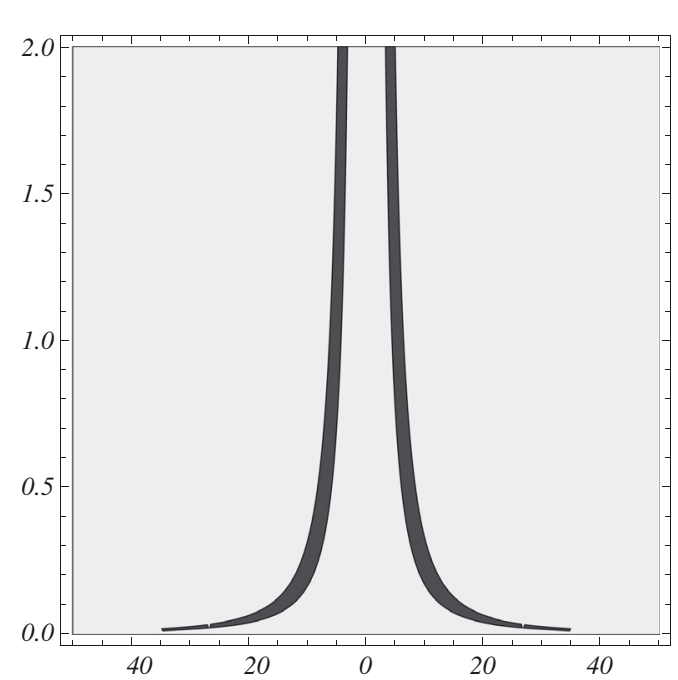

(a) $\rho=10, F$-axis and $A y$-axis

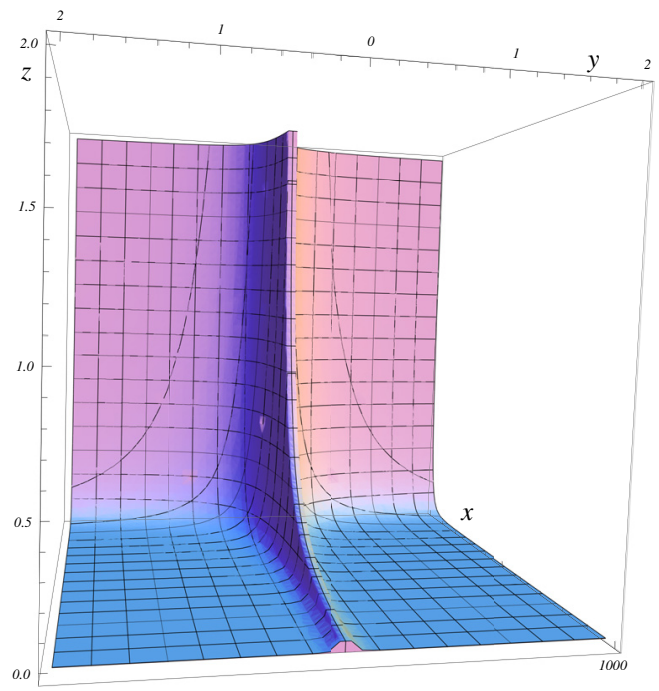

(b) $3 \mathrm{~d}$ hyperbolic region for $: 0 \leqslant \rho \leqslant 1000$ ( $x$-axis), $-2 \leqslant F \leqslant 2$ (y-axis), $0 \leqslant A \leqslant 2$ (z-axis)

FiguRE 5. Strip hyperbolic range and the hyperbolic set.

(2) System (2.10) admits a mathematical total energy

$$
\mathcal{E}=E_{a}+E_{w}
$$

with

$$
E_{a}=\frac{M v^{2}}{2}+\frac{c_{a}^{2} M}{\gamma(\gamma-1)}
$$

and

$$
E_{w}=\frac{A u^{2}}{2}+g A\left(h_{w}-I_{1}(x, A) / A\right) \cos \theta+g A Z
$$

which satisfies, for smooth solutions, the entropy equality

$$
\partial_{t} \mathcal{E}+\partial_{x} \mathcal{H}=0
$$

where the flux $\mathcal{H}$ is the total entropy flux defined by

$$
\mathcal{H}=\mathcal{H}_{a}+\mathcal{H}_{w}
$$

where the air entropy flux reads:

$$
\mathcal{H}_{a}=\left(E_{a}+\frac{c_{a}^{2} M}{\gamma}\right) v
$$

and the water entropy flux reads:

$$
\mathcal{H}_{w}=\left(E_{w}+g I_{1}(x, A) \cos \theta+A \frac{c_{a}^{2} M}{(S-A)}\right) u .
$$


(3) The energies $E_{a}$ and $E_{w}$ satisfy the following entropy flux equalities:

$$
\partial_{t} E_{a}+\partial_{x} \mathcal{H}_{a}=\frac{c_{a}^{2} M}{\gamma(S-A)} \partial_{t} A
$$

and

$$
\partial_{t} E_{w}+\partial_{x} \mathcal{H}_{w}=-\frac{c_{a}^{2} M}{\gamma(S-A)} \partial_{t} A
$$

Proof of Theorem 2.3. The proof of these statements relies only on algebraic combinations of the two equations forming System (2.10).

Remark 2.8. Let us remark that equations (2.14)-(2.15) can be interpreted in such a way: the system is physically closed i.e. the energy dissipated (or gained) by the water layer is forwarded to (or lost by) the air layer. This property ensures the total energy equality

$$
\partial_{t} \mathcal{E}+\partial_{x} \mathcal{H}=0
$$

\section{ThE KINETIC INTERPRETATION OF THE TWO-LAYER AIR-WATER MODEL}

As already mentioned before, the coupling between the layers, due to the coupling of an hydrostatic pressure and a barotropic pressure law, does not provide an explicit access to the eigenstructure of the system, which is required for numerical schemes based on the solution of the Riemann problem. It forms a system which is conditionally hyperbolic (see Thm. 2.2). Nevertheless, one can provide the first order expansions with respect to the relative speed as approximate expressions for the eigenvalues (see for instance [1,2,31]). Let us mention that Abgrall and Karni [1] provides a way to decouple the system and to have an access to the eigenvalues by using a relaxation approach.

In this work, the computation of the eigenvalues is out of interest since we will focus on the kinetic interpretation of the two-layer model. This is a useful way to compute the numerical solution even if the system loses its hyperbolicity since the computation of the eigenvalues are not required. Moreover following $[9,11]$, natural properties are obtained such as the apparition or vanishing of vacuum in the air layer or drying and flooding flows for the water layer. All of these properties will be useful in the future development for more physical models. Although at the numerical level, the effect of the variable cross section is not taken into account, this framework offers an easy way to upwind the source terms and to get well-balanced numerical schemes.

The particular treatment of the boundary conditions will be rapidly exposed using the "decoupled system" approach.

\subsection{The mathematical kinetic formulation}

Let $\chi: \mathbb{R} \rightarrow \mathbb{R}$ be a given real function satisfying the following properties:

$$
\chi(\omega)=\chi(-\omega) \geqslant 0, \int_{\mathbb{R}} \chi(\omega) \mathrm{d} \omega=1, \int_{\mathbb{R}} \omega^{2} \chi(\omega) \mathrm{d} \omega=1 .
$$

It permits to define the density of particles, by a so-called Gibbs equilibrium,

$$
\mathcal{M}_{\alpha}(t, x, \xi)=\frac{A_{\alpha}(t, x)}{b_{\alpha}(t, x)} \chi\left(\frac{\xi-u_{\alpha}(t, x)}{b_{\alpha}(t, x)}\right)
$$

where $b_{\alpha}(t, x)=b(x, A(t, x), M(t, x))$ with

$$
b_{\alpha}^{2}(x, A, M)= \begin{cases}\frac{c_{a}^{2}}{\gamma M} & \text { if } \alpha=a, \\ g \frac{I_{1}(x, A)}{A} \cos \theta+\frac{c_{a}^{2} M}{\gamma(S-A)} & \text { if } \alpha=w,\end{cases}
$$


and

$$
A_{\alpha}=\left\{\begin{array}{ll}
A & \text { if } \alpha=w \\
M & \text { if } \alpha=a
\end{array}, Q_{\alpha}=\left\{\begin{array}{ll}
Q & \text { if } \alpha=w \\
D & \text { if } \alpha=a
\end{array} \text { and } u_{\alpha}=\left\{\begin{array}{ll}
u & \text { if } \alpha=w \\
v & \text { if } \alpha=a
\end{array} .\right.\right.\right.
$$

The Gibbs equilibrium $\mathcal{M}_{\alpha}$ is related to the two-layer model (2.10) by the classical macro-microscopic kinetic relations:

$$
\begin{gathered}
A_{\alpha}=\int_{\mathbb{R}} \mathcal{M}_{\alpha}(t, x, \xi) \mathrm{d} \xi, \\
Q_{\alpha}=\int_{\mathbb{R}} \xi \mathcal{M}_{\alpha}(t, x, \xi) \mathrm{d} \xi, \\
\frac{Q_{\alpha}^{2}}{A_{\alpha}}+A_{\alpha} b_{\alpha}(x, A, M)^{2}=\int_{\mathbb{R}} \xi^{2} \mathcal{M}_{\alpha}(t, x, \xi) \mathrm{d} \xi .
\end{gathered}
$$

From the relations (3.3)-(3.5), the nonlinear two-layer model can be viewed as two single linear equations, one for each layer, involving the nonlinear quantity $\mathcal{M}_{\alpha}$ :

Theorem 3.1 (kinetic formulation of the PFS model). Assuming a constant angle $\theta,(A, Q, M, D)$ is a strong solution of System (2.10) if and only if $\left(\mathcal{M}_{w}, \mathcal{M}_{a)}\right.$ satisfies the system of coupled kinetic transport equations:

$$
\left\{\begin{array}{l}
\partial_{t} \mathcal{M}_{w}+\xi \cdot \partial_{x} \mathcal{M}_{w}-\left(g \partial_{x} Z-g \frac{I_{2}(x, A)}{A} \cos \theta-\frac{M}{\gamma A(S-A)} c_{a}^{2} \partial_{x} A\right) \partial_{\xi} \mathcal{M}_{w}=\mathcal{K}_{w}(t, x, \xi) \\
\partial_{t} \mathcal{M}_{a}+\xi \cdot \partial_{x} \mathcal{M}_{a}-\left(-\frac{c_{a}^{2}}{\gamma(S-A)} \partial_{x}(S-A)\right) \partial_{\xi} \mathcal{M}_{a}=\mathcal{K}_{a}(t, x, \xi)
\end{array}\right.
$$

for some collision term $\mathcal{K}_{w}(t, x, \xi)$ and $\mathcal{K}_{a}(t, x, \xi)$ which satisfies for $(t, x)$ a.e.

$$
\int_{\mathbb{R}}\left(\begin{array}{l}
1 \\
\xi
\end{array}\right) \mathcal{K}_{w}(t, x, \xi) \mathrm{d} \xi=0, \int_{\mathbb{R}}\left(\begin{array}{l}
1 \\
\xi
\end{array}\right) \mathcal{K}_{a}(t, x, \xi) \mathrm{d} \xi=0
$$

Proof. The proof relies on very obvious computations since $\mathcal{M}_{\alpha}$ verifies the macro-microscopic kinetic relations (3.3)-(3.5).

\section{Remark 3.1.}

- In order to write the system (3.6) in a compact form, we use the indexes $w$ for the water and $a$ for the air and we obtain:

$$
\partial_{t} \mathcal{M}_{\alpha}+\xi \cdot \partial_{x} \mathcal{M}_{\alpha}-\phi_{\alpha} \partial_{\xi} \mathcal{M}_{\alpha}=\mathcal{K}_{\alpha}(t, x, \xi)
$$

The source terms $\phi_{\alpha}(x, \boldsymbol{W})$ are defined as:

$$
\phi_{\alpha}(x, \boldsymbol{W})=\boldsymbol{B}_{\alpha}(x, \boldsymbol{W})^{t}\left(\partial_{x} \boldsymbol{W}_{\alpha}\right)
$$

with

$$
\boldsymbol{W}_{\alpha}= \begin{cases}(Z, S, A) & \text { if } \alpha=w \\ \mathcal{A}:=S-A & \text { if } \alpha=a\end{cases}
$$

and $\boldsymbol{B}_{\alpha}= \begin{cases}\left(g,-g \frac{I_{2}(x, A)}{A \partial_{x} S} \cos \theta,-\frac{M}{\gamma A(S-A)} c_{a}^{2}\right) & \text { if } \alpha=w \\ -\frac{c_{a}^{2}}{\gamma(S-A)} & \text { if } \alpha=a .\end{cases}$

- The kinetic formulation presented in Theorem 3.1 is a (non physical) microscopic description of the two layer model (2.10).

- The results of Theorem 3.1 are obtained assuming a constant angle $\theta$. The generalization of these results with varying $\theta=\theta(x)$ can be obtained by adding the term of curvature mentioned in Remark 2.3 as done in $[8,9,11]$, for a single fluid flow. It can be easily adapted to this case. 


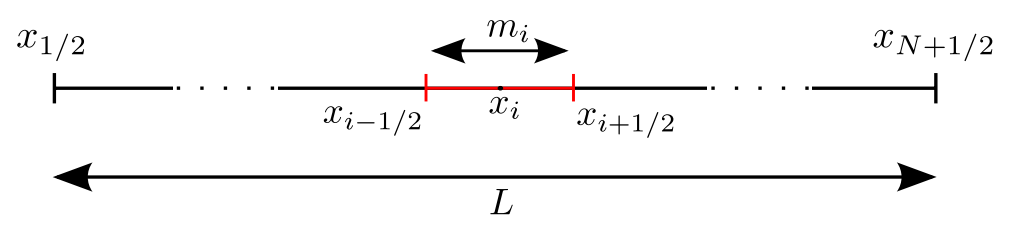

Figure 6. The space discretisation.

\section{THE TWO-LAYER KINETIC SCHEME}

In this section, following the works of $[9,11,26]$, we will construct a two-layer finite volume kinetic scheme that is area conservative, that preserves the posivity of the area and that computes "naturally" flooding, drying and vacuum zones, even when the system loses its hyperbolicity. Although, we are not interested in the treatment of the source term, the full kinetic scheme will be provided.

Under this assumption and based on the kinetic formulation (see Thm. 3.1), we construct easily the two-layer finite volume scheme where the conservative quantities are cell-centered and source terms are included into the numerical fluxes by a standard kinetic scheme with reflections [26].

\subsection{The general formulation}

Let $N \in \mathbb{N}^{*}$, and let us consider the following mesh on $[0, L]$ of a pipe of length $L$. Cells are denoted for every $i \in[0, N+1]$, by $m_{i}=\left(x_{i-1 / 2}, x_{i+1 / 2}\right)$, with $x_{i}=\frac{x_{i-1 / 2}+x_{i+1 / 2}}{2}$ and $h_{i}=x_{i+1 / 2}-x_{i-1 / 2}$ the space step. The "fictitious" cells $m_{0}$ and $m_{N+1}$ denote the boundary cells and the mesh interfaces located at $x_{1 / 2}$ and $x_{N+1 / 2}$ are respectively the upstream and the downstream ends of the pipe (see Fig. 6).

We also consider a time discretization $t^{n}$ defined by $t^{n+1}=t^{n}+\Delta t^{n}$ with $\Delta t^{n}$ the time step.

We denote $\boldsymbol{U}_{\alpha, i}^{n}=\left(A_{\alpha, i}^{n}, Q_{\alpha, i}^{n}\right), u_{\alpha, i}^{n}=\frac{Q_{\alpha, i}^{n}}{A_{\alpha, i}^{n}}, \mathcal{M}_{\alpha, i}^{n}$ the cell-centered approximation of $\boldsymbol{U}_{\alpha}=\left(A_{\alpha}, Q_{\alpha}\right), u_{\alpha}$ and $\mathcal{M}_{\alpha}$ on the cell $m_{i}$ at time $t^{n}$ where we use the notation subscript $\alpha$ introduced previously. We denote by $\boldsymbol{U}_{\alpha, 0}^{n}=\left(A_{\alpha, 0}^{n}, Q_{\alpha, 0}^{n}\right)$ the upstream and $\boldsymbol{U}_{\alpha, N+1}^{n}=\left(A_{\alpha, N+1}^{n}, Q_{\alpha, N+1}^{n}\right)$ the downstream state vectors.

The piecewise constant representation of $\boldsymbol{W}_{\alpha}$ defined by (3.8) is given by, $\boldsymbol{W}_{\alpha}(t, x)=\boldsymbol{W}_{\alpha, i}(t) \mathbb{1}_{m_{i}}(x)$ where $\boldsymbol{W}_{\alpha, i}(t)$ is defined as $\boldsymbol{W}_{\alpha, i}(t)=\frac{1}{\Delta x} \int_{m_{i}} \boldsymbol{W}_{\alpha}(t, x) \mathrm{d} x$ for instance.

Denoting by $\boldsymbol{W}_{\alpha, i}$ and $\boldsymbol{W}_{\alpha, i+1}$ the left hand side and the right hand side values of $\boldsymbol{W}$ at the cell interface $x_{i+1 / 2}$, and using the "straight lines" paths (see [16])

$$
\Psi\left(s, \boldsymbol{W}_{\alpha, i}, \boldsymbol{W}_{\alpha, i+1}\right)=s \boldsymbol{W}_{\alpha, i+1}+(1-s) \boldsymbol{W}_{\alpha, i}, s \in[0,1],
$$

we define the non-conservative product $\phi_{\alpha}\left(t, x_{i+1 / 2}\right)$ by writing:

$$
\phi_{\alpha}\left(t, x_{i+1 / 2}\right)=\left[\left[\boldsymbol{W}_{\alpha}\right]\right](t) \cdot \int_{0}^{1} \boldsymbol{B}\left(t, \Psi\left(s, \boldsymbol{W}_{\alpha, i}(t), \boldsymbol{W}_{\alpha, i+1}(t)\right)\right) \mathrm{d} s
$$

where $\left[\left[\boldsymbol{W}_{\alpha}\right]\right](t):=\boldsymbol{W}_{\alpha, i+1}(t)-\boldsymbol{W}_{\alpha, i}(t)$, is the jump of $\boldsymbol{W}_{\alpha}(t)$ across the discontinuity localized at $x=x_{i+1 / 2}$. As the first component of $\boldsymbol{B}_{w}$ is $g$, we recover the classical interfacial upwinding for the conservative term $Z$ as initially introduced in [26].

Neglecting the collision kernel as in $[9,11,26]$ and using the fact that $\phi_{\alpha}=0$ on the cell $m_{i}$ (since $\boldsymbol{W}_{\alpha}$ is constant on $m_{i}$ ), the kinetic transport equation (3.6) simply reads:

$$
\frac{\partial}{\partial t} \mathcal{M}_{\alpha}+\xi \cdot \frac{\partial}{\partial x} \mathcal{M}_{\alpha}=0 \quad \text { for } x \in m_{i}
$$

This equation is a linear transport equation whose explicit discretization may be done directly by the following way. 
Denoting for $x \in m_{i}, f_{\alpha}\left(t_{n}, x, \xi\right)=\mathcal{M}_{\alpha, i}^{n}(\xi)=\mathcal{M}_{\alpha}\left(A_{\alpha, i}^{n}, Q_{\alpha, i}^{n}, \xi\right)$ the Maxwellian state associated to $A_{\alpha, i}^{n}$ and $Q_{\alpha, i}^{n}$, a finite volume discretization of equation (4.2) leads to:

$$
f_{\alpha, i}^{n+1}(\xi)=\mathcal{M}_{\alpha, i}^{n}(\xi)+\frac{\Delta t}{h_{i}} \xi\left(\mathcal{M}_{\alpha, i+\frac{1}{2}}^{-}(\xi)-\mathcal{M}_{\alpha, i-\frac{1}{2}}^{+}(\xi)\right)
$$

where the fluxes $\mathcal{M}_{\alpha, i+\frac{1}{2}}^{ \pm}$have to take into account the discontinuity of the source term $\phi_{\alpha}$ at the cell interface $x_{i+1 / 2}$. This is the principle of interfacial source upwind. Indeed, noticing that the fluxes can also be written as:

$$
\mathcal{M}_{\alpha, i+\frac{1}{2}}^{-}(\xi)=\mathcal{M}_{\alpha, i+\frac{1}{2}}+\left(\mathcal{M}_{\alpha, i+\frac{1}{2}}^{-}-\mathcal{M}_{\alpha, i+\frac{1}{2}}\right)
$$

the quantity $\delta \mathcal{M}_{\alpha, i+\frac{1}{2}}^{-}=\mathcal{M}_{\alpha, i+\frac{1}{2}}^{-}-\mathcal{M}_{\alpha, i+\frac{1}{2}}$ holds for the discrete contribution of the source term $\phi_{\alpha}$ in the system for negative velocities $\xi \leq 0$ due to the upwinding of the source term. Thus $\delta \mathcal{M}_{\alpha, i+\frac{1}{2}}^{-}$has to vanish for positive velocity $\xi>0$, as proposed by the choice of the interface fluxes below. Let us now detail our choice for the fluxes $\mathcal{M}_{\alpha, i+\frac{1}{2}}^{ \pm}$at the interface. It can be justified by using a generalized characteristic method for equation (3.6) (without the collision kernel) but we give instead a presentation based on some physical energetic balance. The details of the construction of these fluxes by the general characteristics method (see [15], Def. 2.1) is done in [17], Chapter 2.

In order to take into account the neighboring cells by means of a natural interpretation of the microscopic features of the system, we formulate a peculiar discretization for the fluxes in (4.3), computed by the following upwinded formulas:

$$
\begin{aligned}
\mathcal{M}_{\alpha, i+1 / 2}^{-}(\xi)= & \overbrace{\mathbb{1}_{\{\xi>0\}} \mathcal{M}_{\alpha, i}^{n}(\xi)}^{\text {positive transmission }}+\overbrace{\mathbb{1}_{\left\{\xi<0, \xi^{2}-2 g \Delta \phi_{\alpha, i+1 / 2}^{n}<0\right\}} \mathcal{M}_{\alpha, i}^{n}(-\xi)}^{\text {reflection }} \\
+ & \underbrace{\mathbb{1}_{\left\{\xi<0, \xi^{2}-2 g \Delta \phi_{\alpha, i+1 / 2}^{n}>0\right\}} \mathcal{M}_{\alpha, i+1}^{n}\left(-\sqrt{\xi^{2}-2 g \Delta \phi_{\alpha, i+1 / 2}^{n}}\right)}_{\text {negative transmission }}, \\
\mathcal{M}_{\alpha, i+1 / 2}^{+}(\xi)= & \overbrace{\mathbb{1}_{\{\xi<0\}} \mathcal{M}_{\alpha, i+1}^{n}(\xi)}^{\text {negative transmission }}+\overbrace{\mathbb{1}_{\left\{\xi>0, \xi^{2}+2 g \Delta \phi_{\alpha, i+1 / 2}^{n}<0\right\}} \mathcal{M}_{\alpha, i+1}^{n}(-\xi)}^{\text {reflection }}) \\
& +\underbrace{\mathbb{1}_{\left\{\xi>0, \xi^{2}+2 g \Delta \phi_{\alpha, i+1 / 2}^{n}>0\right\}} \mathcal{M}_{\alpha, i}^{n}\left(\sqrt{\xi^{2}+2 g \Delta \phi_{\alpha, i+1 / 2}^{n}}\right)}_{\text {positive transmission }} .
\end{aligned}
$$

The term $\Delta \phi_{\alpha, i \pm 1 / 2}^{n}$ in (4.4) is the upwinded source term (3.7). It also plays the role of the potential barrier: the term $\xi^{2} \pm 2 g \Delta \phi_{\alpha, i+1 / 2}^{n}$ is the jump condition for a particle with a kinetic speed $\xi$ which is necessary to

- be reflected: this means that the particle has not enough kinetic energy $\xi^{2} / 2$ to overpass the potential barrier (reflection term in (4.4));

- overpass the potential barrier with a positive speed (positive transmission term in (4.4));

- overpass the potential barrier with a negative speed (negative transmission term in (4.4)).

Taking an approximation of the non-conservative product $\phi_{\alpha}$ defined by equation (4.1), the potential barrier $\Delta \phi_{\alpha, i+1 / 2}^{n}$ has the following expression:

$$
\Delta \phi_{\alpha, i+1 / 2}^{n}=\left[\left[\boldsymbol{W}_{\alpha}\right]\right]\left(t_{n}\right) \cdot \boldsymbol{B}_{\alpha}\left(t_{n}, \Psi\left(\frac{1}{2}, \boldsymbol{W}_{\alpha, i}\left(t_{n}\right), \boldsymbol{W}_{\alpha, i+1}\left(t_{n}\right)\right)\right)
$$

which is an approximation of $\Delta \phi_{\alpha}$ by the midpoint quadrature formula. 
Since we neglected the collision term, it is clear that $f_{\alpha}^{n+1}$ computed by the discretized kinetic equation (4.3) is no more a Gibbs equilibrium. Therefore, to recover the macroscopic variables $A_{\alpha}$ and $Q_{\alpha}$, according to the identities (3.3)-(3.4), we set:

$$
\boldsymbol{U}_{\alpha, i}^{n+1}=\left(\begin{array}{l}
A_{\alpha, i}^{n+1} \\
Q_{\alpha, i}^{n+1}
\end{array}\right) \stackrel{\text { def }}{=} \int_{\mathbb{R}}\left(\begin{array}{l}
1 \\
\xi
\end{array}\right) f_{\alpha, i}^{n+1} \mathrm{~d} \xi
$$

In fact at each time step, we projected $f_{\alpha}^{n}(\xi)$ on $\mathcal{M}_{\alpha, i}^{n}(\xi)$, which is a way to perform all collisions at once and to recover a Gibbs equilibrium without computing it.

Now, we can integrate the discretized kinetic equation (4.3) against 1 and $\xi$ to obtain the macroscopic kinetic scheme:

$$
\boldsymbol{U}_{\alpha, i}^{n+1}=\boldsymbol{U}_{\alpha, i}^{n}+\frac{\Delta t^{n}}{h_{i}}\left(\boldsymbol{F}_{\alpha, i+\frac{1}{2}}^{-}-\boldsymbol{F}_{\alpha, i-\frac{1}{2}}^{+}\right) .
$$

The numerical fluxes are thus defined by the kinetic fluxes as follows:

$$
\boldsymbol{F}_{\alpha, i+\frac{1}{2}}^{ \pm} \stackrel{\text { def }}{=} \int_{\mathbb{R}} \xi\left(\begin{array}{l}
1 \\
\xi
\end{array}\right) \mathcal{M}_{\alpha, i+\frac{1}{2}}^{ \pm}(\xi) \mathrm{d} \xi
$$

Computing the macroscopic state $\boldsymbol{U}_{\alpha}$ by equations (4.5)-(4.6) is not easy if the function $\chi$ verifying the properties (3.1) is not compactly supported (see [26]).

Moreover, as we shall see in the next proposition, a CFL conditions is needed to obtain a scheme that preserves the positivity of the wet area.

Proposition 4.1. Let $\chi$ be a compactly supported function verifying (3.1) and note $[-K, K]$ its support. The kinetic scheme (4.5)-(4.6) has the following properties:

(1) the kinetic scheme is a $A_{\alpha}$ conservative scheme;

(2) assume the following CFL condition

$$
\Delta t^{n} \max _{\alpha, i}\left(\left|u_{\alpha, i}^{n}\right|+K b_{\alpha, i}^{n}\right) \leqslant \min _{i} h_{i}
$$

holds. Then the kinetic scheme keeps the wet area $A_{\alpha}$ positive i.e.:

$$
\text { if, for every } i \in[0, N+1], A_{\alpha, i}^{0} \geqslant 0 \text { then, for every } i \in[0, N+1], A_{\alpha, i}^{n} \geqslant 0 \text {; }
$$

(3) the kinetic scheme treats "naturally" flooding and drying zones for the water layer, and the apparition of vacuum for the air layer.

Proof. We will adapt the proof of [26] to show the three properties that verify the kinetic scheme.

1. Let us denote the first component of the discrete fluxes $(4.6)\left(F_{A_{\alpha}}\right)_{i+\frac{1}{2}}^{ \pm}$:

$$
\left(F_{A_{\alpha}}\right)_{i+\frac{1}{2}}^{ \pm} \stackrel{\text { def }}{=} \int_{\mathbb{R}} \xi \mathcal{M}_{\alpha, i+\frac{1}{2}}^{ \pm}(\xi) \mathrm{d} \xi
$$

An easy computation, using the change of variable $\mu=|\xi|^{2}-2 g \Delta \phi_{\alpha, i+\frac{1}{2}}^{n}$, allows us to show that:

$$
\left(F_{A_{\alpha}}\right)_{i+\frac{1}{2}}^{+}=\left(F_{A_{\alpha}}\right)_{i+\frac{1}{2}}^{-}
$$


2. Suppose that for every $i \in[0, N+1], A_{\alpha, i}^{n}>0$. Let us note $\xi_{ \pm}=\max (0, \pm \xi)$ and $\sigma=\frac{\Delta t^{n}}{\min _{i} h_{i}}$. From equation (4.3), we get the following inequalities:

$$
\begin{aligned}
f_{\alpha, i}^{n+1}(\xi) \geqslant & (1-\sigma|\xi|) \mathcal{M}_{\alpha, i}^{n}(\xi)+\sigma \xi_{+}\left[\mathbb{1}_{\left\{\xi^{2}+2 g \Delta \phi_{\alpha, i+1 / 2}<0\right\}} \mathcal{M}_{\alpha, i}^{n}(-\xi)\right. \\
& \left.+\mathbb{1}_{\left\{\xi^{2}+2 g \Delta \phi_{\alpha, i-1 / 2}>0\right\}} \mathcal{M}_{\alpha, i-1}^{n}\left(\sqrt{\xi^{2}+2 g \Delta \phi_{\alpha, i+1 / 2}}\right)\right] \\
& +\sigma \xi_{-}\left(\mathbb{1}_{\left\{\xi^{2}-2 g \Delta \phi_{\alpha, i+1 / 2}<0\right\}} \mathcal{M}_{\alpha, i}^{n}(-\xi)\right. \\
& \left.+\mathbb{1}_{\left\{\xi^{2}-2 g \Delta \phi_{\alpha, i-1 / 2}>0\right\}} \mathcal{M}_{\alpha, i+1}^{n}\left(-\sqrt{\xi^{2}-2 g \Delta \phi_{\alpha, i+1 / 2}}\right)\right) .
\end{aligned}
$$

Since the function $\chi$ is compactly supported if $\left|\xi-u_{\alpha, i}^{n}\right| \geqslant K b_{\alpha, i}^{n}$ then $\mathcal{M}_{\alpha, i}^{n}(\xi)=0$. Thus

$$
f_{\alpha, i}^{n+1}(\xi) \geqslant 0 \text { if }\left|\xi-u_{\alpha, i}^{n}\right| \geqslant K b_{\alpha, i}^{n},
$$

as a sum of non negative terms.

On the other hand, for $\left|\xi-u_{\alpha, i}^{n}\right| \leqslant K b_{\alpha, i}^{n}$, using the CFL condition $0<\sigma|\xi| \leqslant 1$, for all $i, f_{\alpha, i}^{n+1} \geqslant 0$ since it is a convex combination of non negative terms.

Finally we have $\forall i \in[0, N+1], f_{\alpha, i}^{n} \geq 0$. Since $\forall i \in[0, N+1], A_{\alpha, i}^{n+1}=\int_{\mathbb{R}} f_{\alpha, i}^{n+1}(\xi) \mathrm{d} \xi$, we finally get $\forall i \in[0, N+1], A_{\alpha, i}^{n+1} \geq 0$.

3. Suppose $A_{i}^{n}=0$. Using the definition of $\mathcal{M}_{w}$, and the fact that the function $\chi$ is compactly supported, the only term that may cause problem is $\frac{A}{b_{w}(t, x)}$. But since

$$
\frac{A}{b(t, x)} \sim \sqrt{\frac{A}{g I_{1}(x, A) \cos \theta}} \quad \text { when } \quad A \sim 0,
$$

we get easily for all the usual pipe geometries (rectangular, trapezoidal, circular, ...):

$$
\lim _{\substack{A \rightarrow 0 \\ A \geq 0}} \frac{A}{b_{w}(t, x)}=0
$$

thus $\mathcal{M}_{w, i}^{n}(\xi)=0$ otherwise we add this as a geometric assumption. This is the reason why we say that the kinetic scheme treats "naturally" the drying and flooding zones. In the same way, for the air layer, the only term which may pose problem is $\frac{M}{b_{a}}$ which is, when $M$ goes to 0 , equivalent to $M^{5 / 2-\gamma}$ where $5 / 2-\gamma=11 / 10>1$. Therefore, we say that the numerical scheme computes "naturally" the apparition/vanishing of vacuum.

Let us finally point out that the $\chi$ function could be chosen undifferently.

\subsection{The "decoupled boundary conditions"}

Without loss of generality, we consider in what follows the case of a uniform section with $\theta=0$ slope. Let us recall that $x_{1 / 2}$ and $x_{N+1 / 2}$ are respectively the upstream and the downstream ends of the pipe. At this stage, we have computed all the "interior" states at time $t^{n+1}$, that is $\left(\boldsymbol{W}_{i}^{n+1}\right)_{i=1, N}:=\left(M_{i}^{n+1}, D_{i}^{n+1}, A_{i}^{n+1}, Q_{i}^{n+1}\right)_{i=1, N}$ are computed.

The upstream state $\boldsymbol{W}_{0}$ corresponds to the mean value of $(M, D, A, Q)$ on the "fictitious" cell $m_{0}=$ $\left(x_{-1 / 2}, x_{1 / 2}\right)$ (at the left of the upstream boundary of the pipe) and the downstream state $\boldsymbol{W}_{N+1}$ corresponds to the mean value of $(M, D, A, Q)$ on the "fictitious" cell $m_{N+1}=\left(x_{N+1 / 2}, x_{N+3 / 2}\right)$ (at the right of the downstream end of the pipe). 
Usually, we have to prescribe at least two boundary conditions related to the state vectors $\boldsymbol{W}_{0}^{n}$ and $\boldsymbol{W}_{N+1}^{n}$. There is generically, that is to say for supercritical flows, "two incoming characteristics curve" for the upstream and two for outgoing characteristics for the downstream when the eigenstructure is known which is clearly not the case in the full system (2.10).

In order to find a complete state for the upstream boundary, $\boldsymbol{W}_{0}^{n+1}$, and the downstream boundary, $\boldsymbol{W}_{N+1}^{n+1}$, in a consistent way, following the recent work of Bourdarias et al. [11], one can use the so-called "kinetic boundary conditions" for which the eigenvalues are not required and can be applied straightforwardly (at the present stage, such boundary conditions are not yet implemented). We present instead a new approach, based on the solution of a linearized Riemann problem with source term, which introduces the concept of "decoupled boundary conditions". Indeed, the full eigenstructure is not available for the full system but dealing with each layers separatively, we have two systems which write:

$$
\partial_{t} \boldsymbol{U}+\mathcal{D}_{w}(\boldsymbol{W}) \partial_{x} \boldsymbol{U}=\left(\begin{array}{c}
0 \\
a c_{a}^{2} \partial_{x} M
\end{array}\right)
$$

and

$$
\partial_{t} \boldsymbol{V}+\mathcal{D}_{a}(\boldsymbol{W}) \partial_{x} \boldsymbol{V}=\left(\begin{array}{c}
0 \\
-c_{a}^{2} \frac{A M}{(S-A)^{2}} \partial_{x} A
\end{array}\right)
$$

where $\boldsymbol{U}=(A, Q)^{t}, \boldsymbol{V}=(M, D)^{t}, \mathcal{D}_{w}(\boldsymbol{W})=\left(\begin{array}{cc}0 & 1 \\ c_{m}^{2}-u^{2} & 2 u\end{array}\right)$ and $\mathcal{D}_{a}(\boldsymbol{W})=\left(\begin{array}{cc}0 & 1 \\ c_{a}^{2}-v^{2} & 2 v\end{array}\right)$ for which eigenstructure is well known and reads as follows for the water layer

$$
\lambda_{w}^{1}(\boldsymbol{W})=u-c_{m}, \quad \lambda_{w}^{2}(\boldsymbol{W})=u+c_{m},
$$

and for the air layer:

$$
\lambda_{a}^{1}(\boldsymbol{W})=v-c_{a}, \quad \lambda_{a}^{2}(\boldsymbol{W})=v+c_{a} .
$$

In theses equations, $u=Q / A$ and $v=D / M$ stands for the mean water and air speed.

Thus, one can apply the usual procedure [17], Chapter 2, (see, also [11]) which consists to assume that the inflow is subcritical, i.e. we have to prescribe at least two boundary conditions one for each layers corresponding to the "two incoming decoupled characteristics curve" for the upstream and two for outgoing characteristics for the downstream. Therefore, for instance, at the upstream end of the pipe, one of the following boundary conditions may be prescribed for the water layer (as usual) (we omit the index $n+1$ for the sake of clarity):

(1) the water level is prescribed. So let $H_{\text {up }}(t)$ be a given function of time. Then we have:

$$
\forall t>0, \quad \mathcal{H}\left(A_{0}(t)\right) \cos \theta_{0}+\mathbf{Z}_{\mathbf{0}}=H_{\text {up }}(t)
$$

(2) the discharge is prescribed. So let $Q_{\mathrm{up}}(t)$ be a given function of time. Then we have:

$$
\forall t>0, \quad Q_{0}(t)=Q_{\mathrm{up}}(t) .
$$

Similarly for the air layer, to be consistent with respect to the previous boundary conditions, we prescribe the air density at the upstream and downstream end. Thus, for instance, if $\left(\rho_{\text {up }}(t), A_{\text {up }}(t)\right)$ are given, by definition $M_{\text {up }}(t):=\frac{\rho_{\text {up }}(t)}{\rho_{0}}\left(S-A_{\text {up }}(t)\right)$ is given and we have to compute $D_{\text {up }}$ and $Q_{\text {up }}$. In the same way, if $\left(\rho_{\text {up }}(t), Q_{\text {up }}(t)\right)$ are given then we have to compute $D_{\text {up }}$ and $A_{\text {up }}$. Once $A_{\text {up }}(t)$ is computed, one has $M_{\text {up }}(t):=\frac{\rho_{\text {up }}(t)}{\rho_{0}}\left(S-A_{\text {up }}(t)\right)$ by definition. For each cases, we have to define two equations, which can be obtained by the usual procedure which consists to solve two linearized Riemann problems for each layers, which can be written into a generic form as follows:

$$
\left\{\begin{array}{l}
\partial_{t} \boldsymbol{U}_{\alpha}+\widetilde{\mathcal{D}_{w}} \partial_{x} \boldsymbol{U}_{\alpha}=G_{\alpha}(\boldsymbol{W}) \\
\boldsymbol{U}_{\alpha}\left(x, t_{n}\right)= \begin{cases}\boldsymbol{U}_{\alpha, 0}^{n} & \text { if } x<0 \\
\boldsymbol{U}_{\alpha, 1}^{n} & \text { if } x>0\end{cases}
\end{array}\right.
$$


where $\widetilde{\mathcal{D}_{\alpha}}$ stands for the constant matrix $\mathcal{D}_{\alpha}(\widetilde{\boldsymbol{W}})$ with $\widetilde{\boldsymbol{W}}=\frac{\boldsymbol{W}_{0}^{n}+\boldsymbol{W}_{1}^{n}}{2}, G_{\alpha}(\boldsymbol{W})$ the source term appearing in equation (4.8) or (4.9) where $\alpha$ stands for the subscript $a$ or $w$. The previous Riemann problem allows to define the missing two equations required to close the system of two unknowns according to the prescribed conditions, and we deduce the two missing equations which read:

$$
\left(Q_{0}^{n+1}-Q_{1}^{n}\right)-\left(A_{0}^{n+1}-A_{1}^{n}\right)\left(\widetilde{u}+\widetilde{c_{m}}\right)=\frac{\Delta t}{2}\left(\left(c_{a}^{2} \frac{A}{S-1}\right)_{0}^{n+1}+\left(c_{a}^{2} \frac{A}{S-1}\right)_{1}^{n}\right)\left(M_{0}^{n+1}-M_{1}^{n}\right)
$$

and

$$
\left(D_{0}^{n+1}-D_{1}^{n}\right)-\left(M_{0}^{n+1}-M_{1}^{n}\right)\left(\widetilde{v}+\widetilde{c_{a}}\right)=\frac{\Delta t}{2}\left(\left(c_{a}^{2} \frac{M}{S-A}\right)_{0}^{n+1}+\left(c_{a}^{2} \frac{M}{S-A}\right)_{1}^{n}\right)\left(A_{0}^{n+1}-A_{1}^{n}\right) .
$$

Let us remark that, when $\left(\rho_{\text {up }}(t), A_{\text {up }}(t)\right)$ are prescribed $Q_{0}^{n+1}$ and $D_{0}^{n+1}$ are respectively explicitly provided by equation (4.13) and (4.14). By the contrary, if $\left(\rho_{\text {up }}(t), Q_{\text {up }}(t)\right)$ are prescribed, in order to get $A_{0}^{n+1}$ and $D_{0}^{n+1}$, we solve the non linear system formed by equation (4.13) and (4.14).

Since the subcritical flows is the only one for which we can write "real boundary conditions", we process by a priori, i.e. after the computation of the subcritical flows as described by the above-mentioned process, we check the validity by computing the sound speed. If such assumption is incorrect, we then switch to the two following configurations:

- an incoming supcritical flows is observed then the critical flows is prescribed (to complete the missing equation);

- an outgoing supcritical flows is observed then we prescribe a free boundary.

We apply such a priori process to deal with such inflow and outflow conditions.

Finally, let us point out that, in previous works the authors have shown its efficiency by validating the treatment of boundary conditions through different test cases in the context of transient mixed water flows in closed water pipes (see for instance $[9,11]$ ).

\section{Numerical tests}

The numerical experiments consist in studying the air entrainment effect in a closed pipe. Since experimental data for such flows in any pipes are not available, we focus only on the qualitative behavior of the proposed model and the presented method for different upstream and downstream conditions.

For all tests, we assume that the air is injected inside the pipe under consideration in order to ensure a constant density of air at upstream and downstream end. For this purpose, we set the upstream and downstream air density $\rho$ to $\rho_{a}$ : we have chosen $\rho_{a}=1.2934910^{-2} \mathrm{~kg} / \mathrm{m}^{3}$ and $\rho_{a}=1.29349 \mathrm{~kg} / \mathrm{m}^{3}$ and as an initial air density, we always set $\rho_{\text {init }}(x)=\rho_{a}$. Then, the unknown state vector $(A, Q, M, D)$ at the upstream and downstream end evolves as described in Section 4.2. We also represent the non hyperbolic region. Finally, at the end of the section, we present a qualitative experiment with respect to the sensitivity of the numerical study with respect to the spatial mesh size when the system is mainly non hyperbolic.

For all tests, the function $\chi$ satisfying (3.1) is defined by:

$$
\chi=\frac{1}{2 \sqrt{3}} \mathbb{1}_{[-\sqrt{3}, \sqrt{3}]} .
$$

\subsection{Non constant upstream boundary conditions}

We consider an horizontal pipe of circular cross-section of $2 \mathrm{~m}^{2}$ is $10 \mathrm{~m}$ long. The altitude of the upstream end of the pipe is $0 \mathrm{~m}$. We start the numerical simulation with a steady state with the water height

$$
h_{\text {init }}(x)=0.05 \mathrm{~m}
$$


(i.e. $\left.A_{\text {init }}(x)=2.0 \times 10^{-2} \mathrm{~m}^{2}\right), M_{\text {init }}(x):=\frac{\rho_{a}}{\rho_{0}}\left(S(x)-A_{\text {init }}(x)\right)=4.0 \times 10^{-3} \mathrm{~m}^{2}$, the water discharge $Q_{\text {init }}(x)=$ $0 \mathrm{~m}^{3} / \mathrm{s}$, the air discharge

$$
D_{\text {init }}(x)=0 \mathrm{~m}^{3} / \mathrm{s},
$$

for all $x \in[0,10]$. The upstream water height is set to

$$
h_{0}(t)=0.05(1+t) \mathbb{1}_{[0,5]}(t)+0.3 \mathbb{1}_{(5,50]}(t)
$$

while the downstream water discharge is kept constant equal to

$$
D_{N+1}(t)=0 \mathrm{~m}^{3} / \mathrm{s} .
$$

These boundary conditions are chosen such that the air-layer model will lose its hyperbolicity for the three following numerical experiments:

(1) the free surface system (called thereafter "single fluid");

(2) the two-layer system with $\rho_{a}=1.2934910^{-2} \mathrm{~kg} / \mathrm{m}^{3}$ fixed at upstream and downstream end;

(3) the two-layer system with $\rho_{a}=1.29349 \mathrm{~kg} / \mathrm{m}^{3}$ fixed at upstream and downstream end.

The single fluid case is solved by a kinetic scheme $[9,11,26]$ while the others tests by the presented "twolayer kinetic scheme". The obtained results are compared in Figures 7-9 at upstream end, at $x=5 \mathrm{~m}$ and at downstream end. We also represents the non hyperbolic region by square points.

Other parameters are:

$\begin{array}{ll}\text { Discretisation points: } & 100, \\ \text { Delta } x(\mathrm{~m}): & 0.1, \\ \text { CFL: } & 0.95, \\ \text { Simulation time (s) : } & 50 .\end{array}$

The case of the so-called "single fluid" has been recently validated through several numerical experiments (see $[9,11])$. Since we use the same methodology, the two-layer kinetic scheme presented here will be compared to the "single fluid" case to show the effect of air entrainment. The presented first numerical experiments simulate the propagation of an hydraulic bore emerging from the upstream end as we increase the upstream water height (see Fig. 7a for $t \leqslant 5 \mathrm{~s}$ ). Then, it reaches the downstream end at, approximatively, $t=7 \mathrm{~s}$ the first time (see Fig. 9a) and it is reflected since the dowstream discharge is kept constant equal to zero. Thereafter, it comes back to the upstream end at, approximatively, $t=17 \mathrm{~s}$ and until $t \approx 27 \mathrm{~s}$ a free boundary conditions is prescribed (which corresponds to an outgoing subcritical flows, i.e. $u_{0}<-c_{0}$, see Figure 7 a and Section 4.2 for further details). This wave propagates alternatively from upstream to downstream before to be damped which is observed in Figures 8a and 9a for $t \geqslant 17 \mathrm{~s}$.

Now, let us focus on the behavior of the numerical solution when the air is taken into account and let's compare the numerical solution with the one obtained for the "single fluid".

As a first remark, let us point out that the shape of the numerical solution of the two-layer system is similar up to a non linear transformation: the shape is rescaled with a higher amplitude. It means that the propagation of the hydraulic bore is also observed when air is taken into account but with a greater celerity: this means that the hydrodynamic behavior of the water is slightly and sharply modified (following the value of $\rho_{a}$ ) under the interaction of air. Globally, in Figures 7-9, this fact is clearly visible when $\rho_{a}=1.2934910^{-2} \mathrm{~kg} / \mathrm{m}^{3}$ is prescribed while we have to pay our attention to the first seconds when $\rho_{a}=1.29349 \mathrm{~kg} / \mathrm{m}^{3}$. For instance, in Figure $8 \mathrm{~b}$, the shape for $t \leqslant 30 \mathrm{~s}$ of the numerical solution of the single fluid has to be compared with the shape for $t \leqslant 8 \mathrm{~s}$ in this case.

As a second remark, let us point that the celerity of this phenomenon is an increasing function of $\rho_{a}$. For instance, when $\rho_{a}=1.29349 \mathrm{~kg} / \mathrm{m}^{3}$, the hydraulic bore reaches immediately the downstream end with a higher amplitude than the case when $\rho_{a}=1.2934910^{-2} \mathrm{~kg} / \mathrm{m}^{3}$ is prescribed which approximatively requires $7 \mathrm{~s}$ (see for example Fig. 9a). These phenomena are the consequence of the prescribed boundary conditions and above 


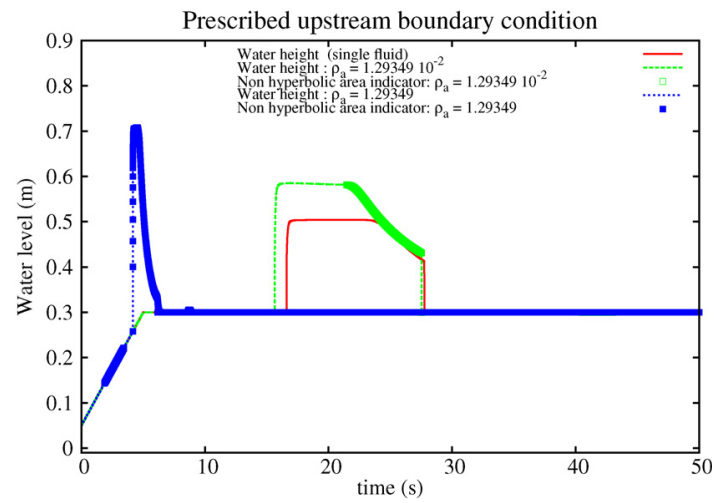

(a) Prescribed water height

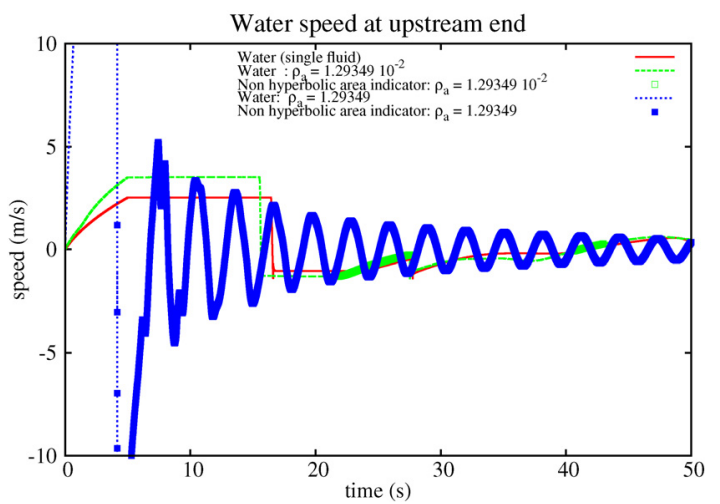

(b) Water speed

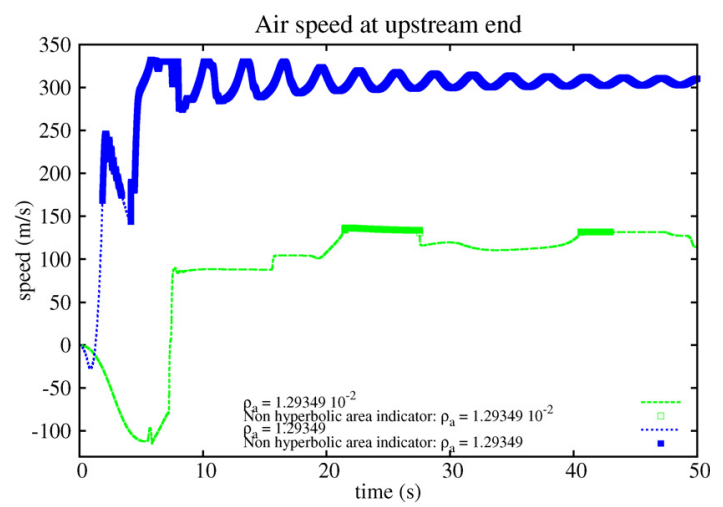

(c) Air speed

FigURE 7. Non constant upstream boundary conditions: numerical results at upstream end.

all of the compressibility of the air layer which interacts with the water layer both confined in a closed area. As the effect are stronger in the case (2) than in the case (3), from now on we focus only in the test case (3).

At time $t=0 \mathrm{~s}$, the air layer as well as the water layer are both in a closed environment. Between, $0 \leqslant t \leqslant 1$, increasing the water height increases the water speed and the air escapes from the upstream (i.e. with a negative speed) as well as in the whole pipe (see Figs. 7c, 8c and 9c) due to the prescribed boundary conditions. Indeed, we increase the water level at upstream end and we set the water discharge to be 0 at downstream end. Therefore, the air necessary escapes throughout the upstream end. After this time period, see Figures $7 \mathrm{~b}$ and $7 \mathrm{c}$ for $1 \leqslant t \leqslant 5$, the air speed reaches a minimum value at $t \approx 1 \mathrm{~s}$ and since we are forcing the air density to be a constant, the upstream boundary equation (4.14) ensures that the upstream air incomes is enough to keep it constant. Consequently, the water speed is again accelerated and we observe a maximum value at $t \approx 5 \mathrm{~s}$. An the same time, as the water flow is accelerated, the hydraulic bore at $t \approx 5 \mathrm{~s}$, after being reflected at the downstream end, is present at the upstream end with a negative speed. This explains why the water speed becomes negative. These phenomena is then reproduce with attenuation of the amplitude of the wave as represented by the oscillation in Figures $7 \mathrm{~b}$ and $7 \mathrm{c}$. The same explanation holds at $x=5 \mathrm{~m}$ and at the downstream end as we observe in Figures $8 \mathrm{~b}, 8 \mathrm{c}, 7 \mathrm{~b}$ and $7 \mathrm{c}$.

These phenomena are reproduced many times, as far as, the initial waves propagates from the upstream to the downstream end until it vanishes due to some dissipative effects. These corresponds to the oscillations observed in Figures 7-9 for the test case (3). 


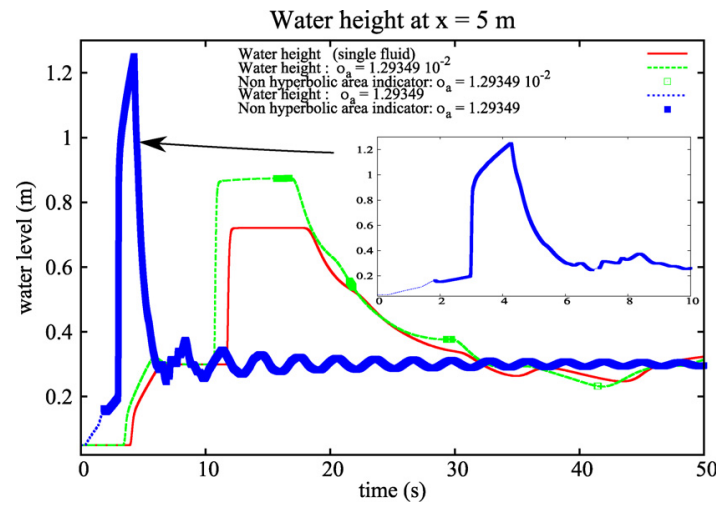

(a) Water height

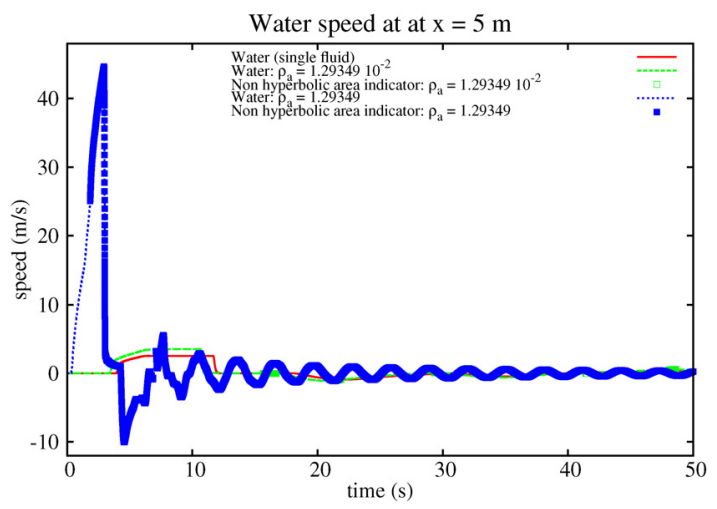

(b) Water speed

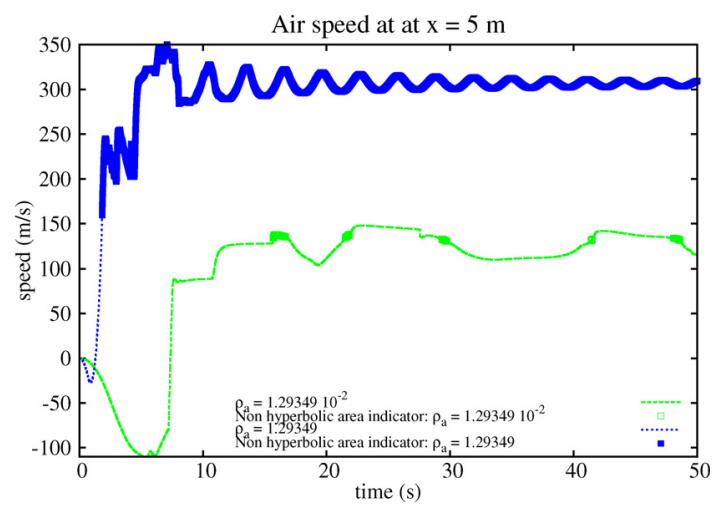

(c) Air speed

FiguRE 8. Non constant upstream boundary conditions: numerical results at $x=5 \mathrm{~m}$.

From now on, let us focus on the non hyperbolic aspect and let us emphasize that the two-layer system loses its hyperbolicity in both tests (2) and (3) and the area of the non hyperbolic region is increasing with respect to $\rho_{a}$ as we can observe in Figures 7-9 (which numerically confirms the analysis done in Sect. 2.3, see Thm. 2.3).

Let us point out that the oscillatory phenomenon is not the so-called geometric growing instabilities and thus not at all related to the loss of hyperbolicity of the two-layer system. Indeed, on one hand, such oscillations are also present for the "single fluid" case but with very small amplitude as we can observe in Figure 9a. On the other hand, in view of the previous explanations of the origin of the oscillations, these phenomena have to be compared with the so-called waterhammer occurring during the filling of the pipe (see, for instance $[7,11]$ ).

These phenomena seems to be a "airhammer" for which the number of oscillations increases with the density of the air (as in the case of pressurized flows with the sound speed, see $[7,11]$ ). This is thus a dynamic consequence of the compressibility of the air layer.

\subsection{Non constant downstream water discharge}

A second numerical tests is done in order to confirm the previous conclusion only for $\rho_{a}=1.29349 \mathrm{~kg} / \mathrm{m}^{3}$ fixed at upstream and downstream end (since the other cases do not show real differences) that we compare to the single fluid in the following settings: the horizontal pipe of circular cross-section of $2 \mathrm{~m}^{2}$ is $100 \mathrm{~m}$ long. The altitude of the upstream end of the pipe is $0 \mathrm{~m}$. We start the numerical simulation with a steady state with the water height $h_{\text {init }}(x)=0.05 \mathrm{~m}$ (i.e. $\left.A_{\text {init }}(x)=2.0 \times 10^{-2} \mathrm{~m}^{2}\right), M_{\text {init }}(x):=\frac{\rho_{a}}{\rho_{0}}\left(S(x)-A_{\text {init }}(x)\right)=4.00 \times 10^{-3} \mathrm{~m}^{2}$, 


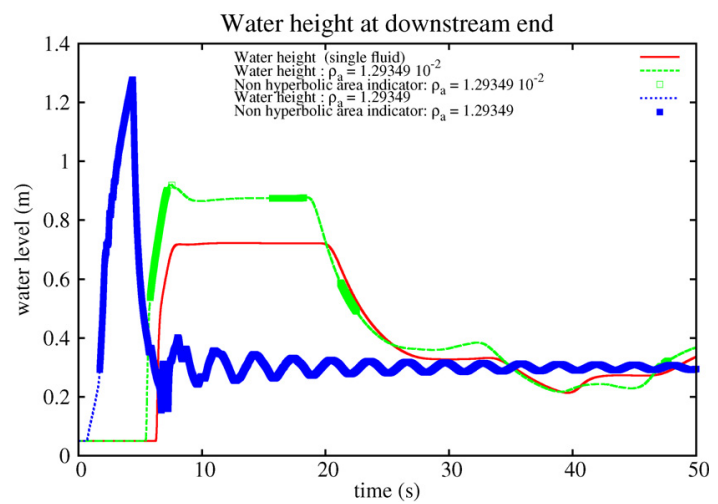

(a) Water height

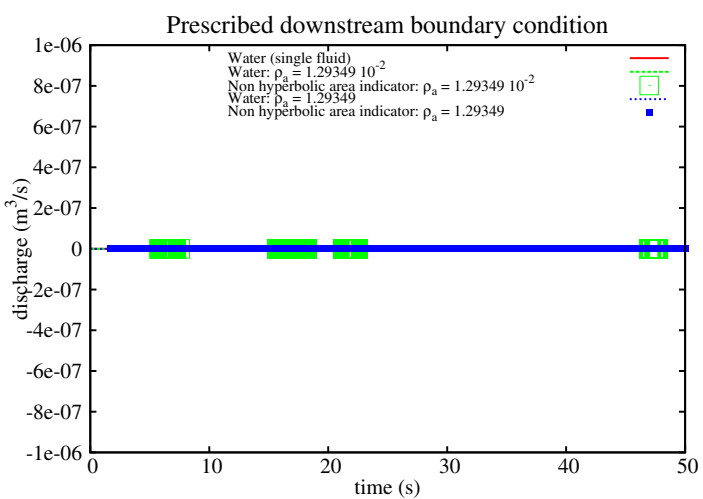

(b) Prescribed downstream discharge

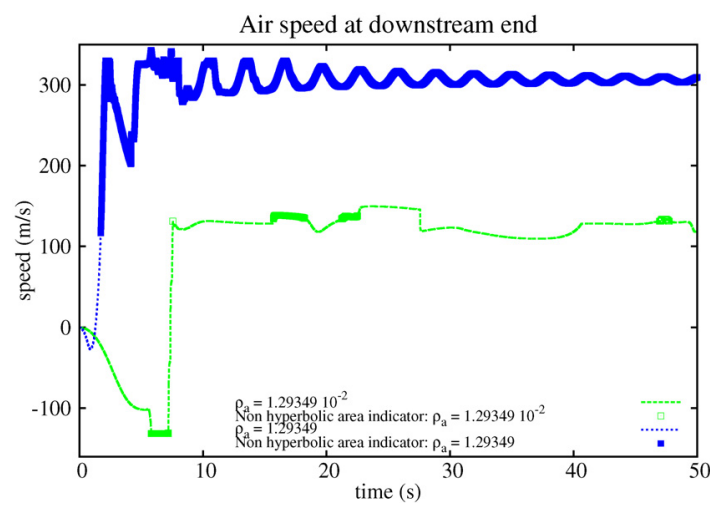

(c) Air speed

FiguRE 9. Non constant upstream boundary conditions: numerical results at downstream end.

the water discharge $Q_{\text {init }}(x)=0 \mathrm{~m}^{3} / \mathrm{s}$, the air discharge $D_{\text {init }}(x)=0 \mathrm{~m}^{3} / \mathrm{s}$, for all $x \in[0,100]$. The upstream water discharge is keep constant to $Q_{0}(t)=0 \mathrm{~m}^{3} / \mathrm{s}$ and the downstream water discharge is set to $Q_{N+1}(t)=-5 t \mathbb{1}_{[0,20]}(t)$. Other parameters are:

\section{Discretisation points: 50 , \\ Delta $x(\mathrm{~m}): \quad 0.5$, \\ CFL: $\quad 0.95$, \\ Simulation time (s): 500 .}

In Figure 10, we represent the water height, the water and air discharge. The same phenomea are reproduced also in the present numerical experiment, i.e. acceleration phenomenon and oscillations are also present. Thus, this confirm the previous observations about such phenomena.

\subsection{Numerical order of the discretization error of the kinetic numerical scheme}

In the previous numerical experiments, we have shown through several examples that the scheme even if the system is not hyperbolic seems to be stable. We have also pointed out that the presence of oscillations was not due to the loss of hyperbolicity. Now, we will check the sensitivity of the two-layer kinetic scheme with respect to decreasing spatial mesh size and show that no geometrically (as we may also emphasize in the previous numerical tests) growing instabilities are observed. 


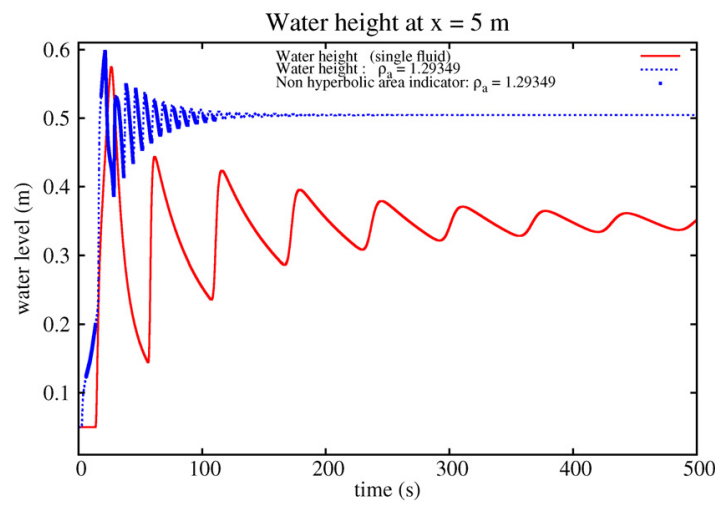

(a) Water height

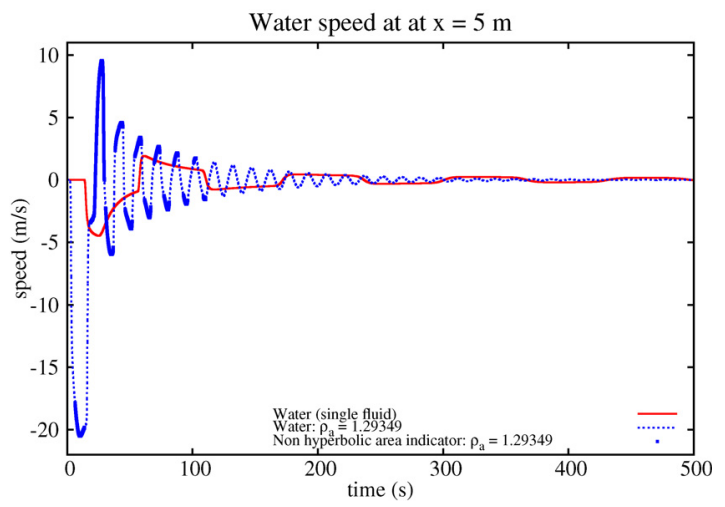

(b) Water speed

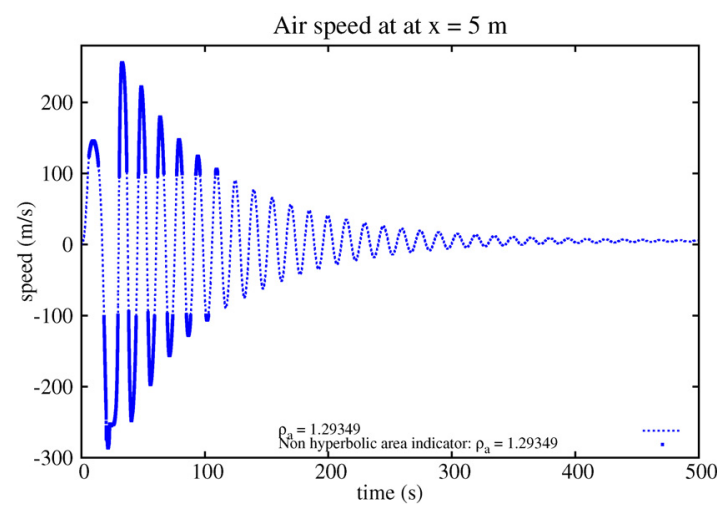

(c) Air speed

FiguRE 10. Non constant downstream water discharge: numerical results at $x=5 \mathrm{~m}$.

In order to obtain a qualitative behavior of the scheme and to compute a "numerical" order of the discretization error of the two-layer kinetic numerical scheme, we present the "dam-break" problem which is defined as initial state by for all $x \in[0, L]$,

$$
Q_{\text {init }}(x)=0 \mathrm{~m}^{3} / \mathrm{s}, \text { and } \quad h_{\text {init }}(x)=1 \mathbb{1}_{[0, L / 2)}(x)+0.5 \mathbb{1}_{(L / 2, L]}(x) \mathrm{m}
$$

for the water layer and $D_{\text {init }}(x)=0 \mathrm{~m}^{3} / \mathrm{s}$ and $M_{\text {init }}(x)=\rho_{\text {init }}(x) / \rho_{0}\left(S-A_{\text {init }}(x)\right)$ for the air layer. We prescribe at the upstream and the downstream end a null water discharge while boundary conditions will be provided by equation (4.14) to compute $D_{0}^{n}$ and $D_{N+1}^{n}$ for each time $t_{n}$.

The horizontal pipe is a circular one of diameter $2 \mathrm{~m}$ and $L=10 \mathrm{~m}$ long. The altitude of the upstream end of the pipe is $0 \mathrm{~m}$. The upstream and downstream discharge is kept constant equal to $0 \mathrm{~m}^{3} / \mathrm{s}$. The air density is chosen such as the two-layer system is mainly non hyperbolic as we can see in Figure 11a: it will be set to $\rho_{a}=1.29349 \mathrm{~kg} / \mathrm{m}^{3}$. We have computed the "exact" numerical flow for all time from $t=0 \mathrm{~s}$ until $t=2 \mathrm{~s}$ with a uniform discretization of $N=1024$ mesh points. We have then computed the $L^{2}$ norm of the difference between the water level computed by the numerical kinetic scheme for different value of the mesh points and the "exact" numerical solution at time $t=0.02 \mathrm{~s}, t=0.2 \mathrm{~s}$ and $t=1.688 \mathrm{~s}$. Results are then compared to the "single fluid" case. 


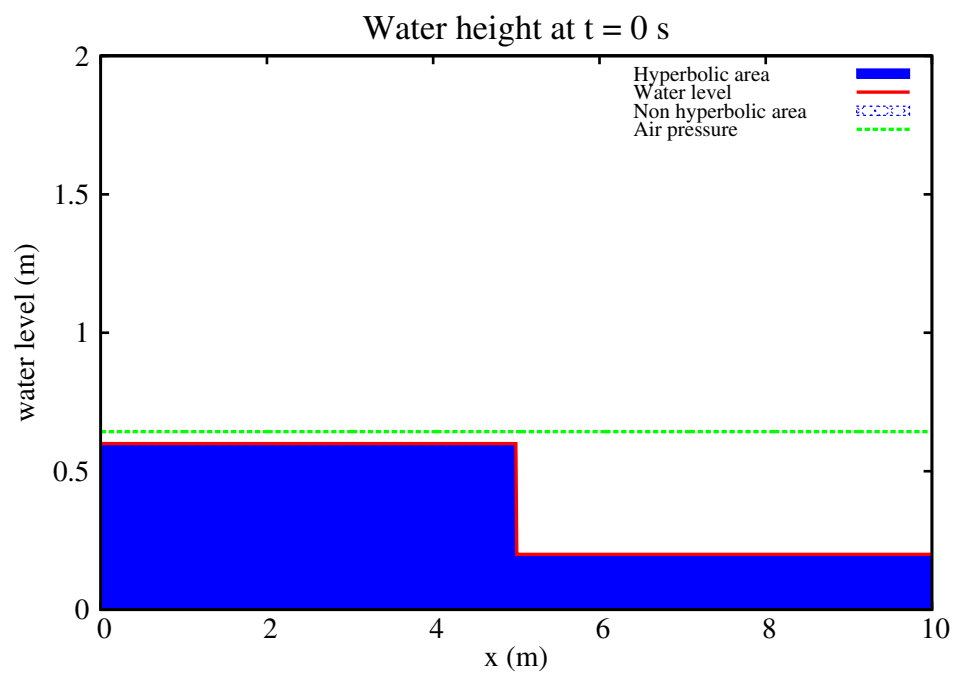

(a) Initial state

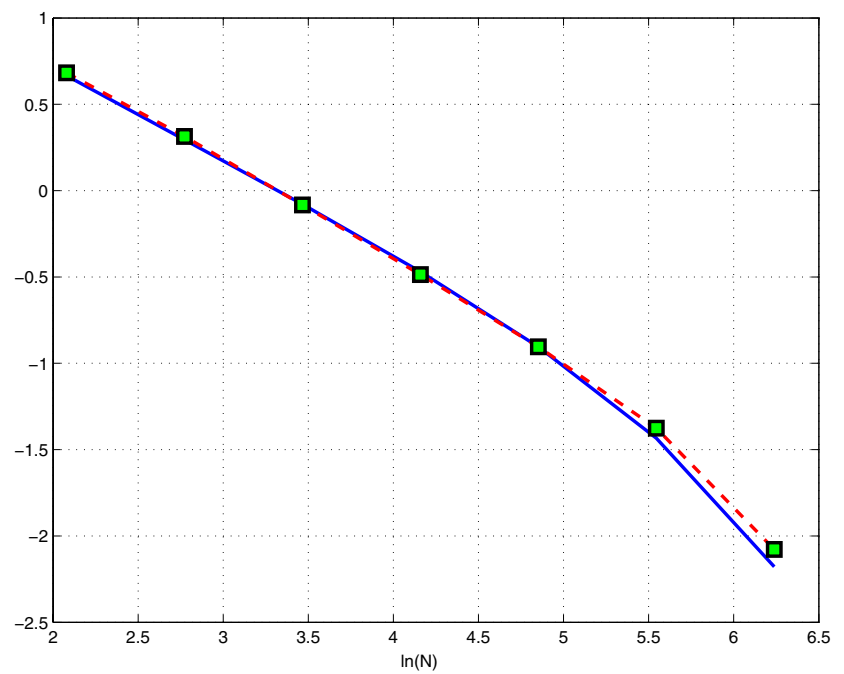

(b) $L_{t}^{1}\left(L_{x}^{2}\right)$ norm of the water level

Figure 11. Initial state for the "dam-break" and the $L_{t}^{1}\left(L_{x}^{2}\right)$ norm of the error (in log scale).

Other parameters are:

CFL:

0.95

Simulation time (s): 2 ,

Delta $t(\mathrm{~s}): \quad 10^{-5}$.

We have fixed the time step in order to obtain the solution of the "single fluid" and two layer model at the same time. Thus, we ensure that $\Delta t=10^{-5}$ satisfies the condition $10^{-5}>\frac{C F L \Delta x}{\max _{\alpha, i=0, \ldots, N+1}\left(\left|u_{\alpha, i}^{n}\right|+\sqrt{3} b_{\alpha, i}^{n}\right)}$.

For each time $t=0.02$ (see Fig. 12), $t=0.2$ (see Fig. 13) and $t=1.688$ (see Fig. 14), we display the shape of the water level for both cases and the obtained $L^{2}$ numerical order. Figure 12 displays the case where the 


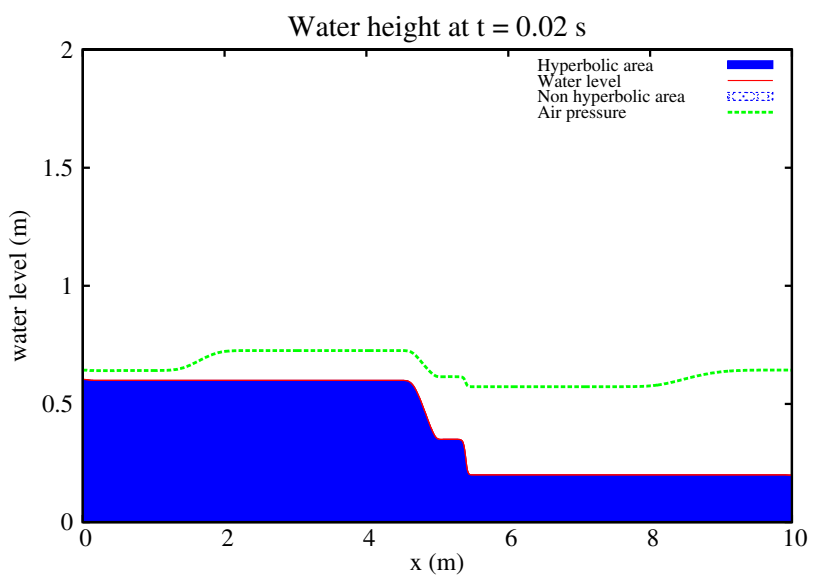

(a) Two-layer model

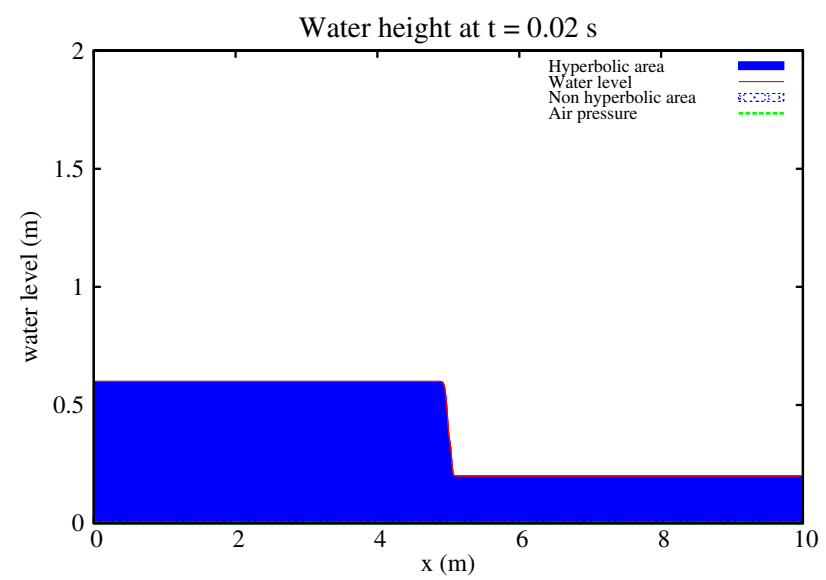

(b) The single fluid

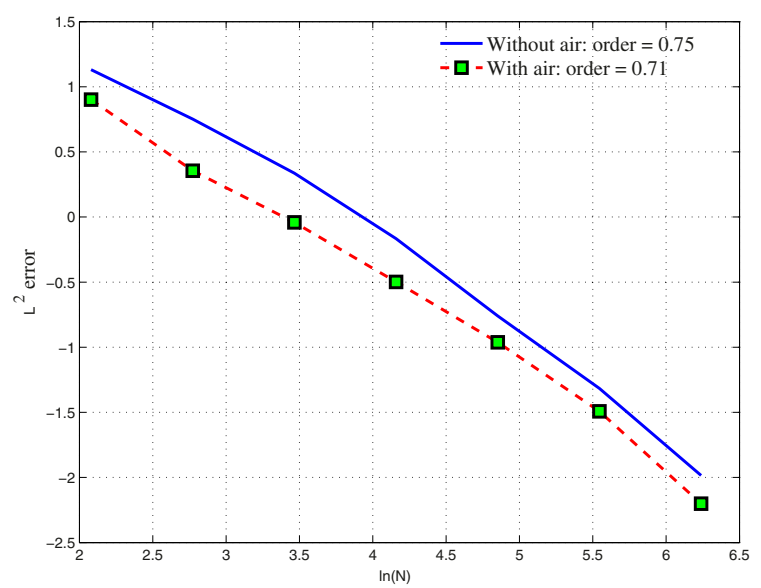

(c) Numerical order

Figure 12. The "dam-break" problem at $t=0.02 \mathrm{~s} . L^{2}$ norm of the error (in log scale). 


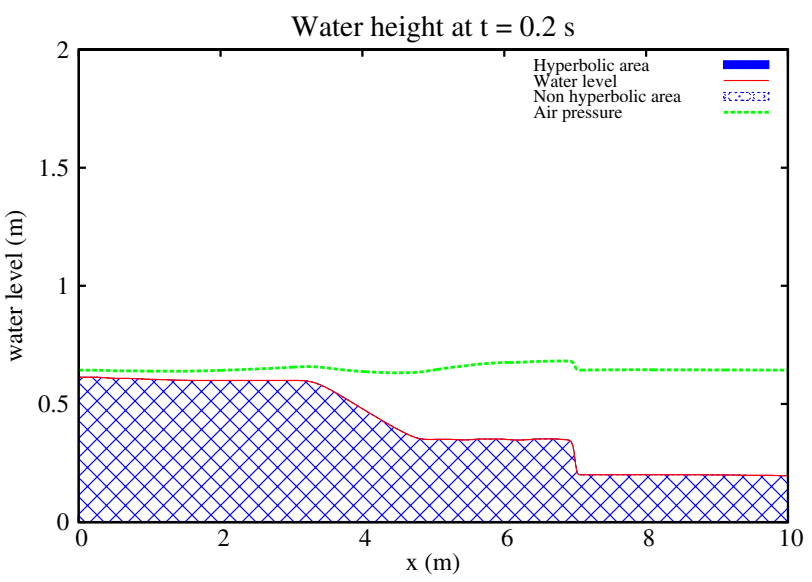

(a) Two-layer model

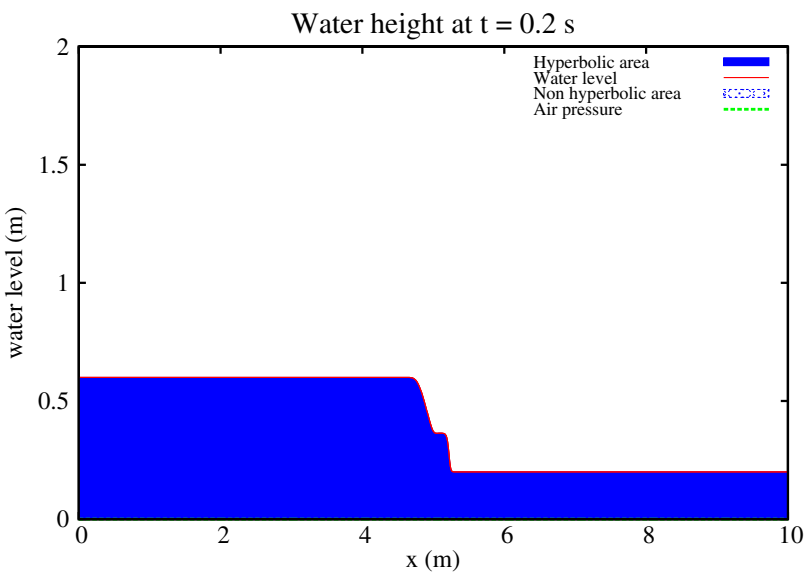

(b) The single fluid

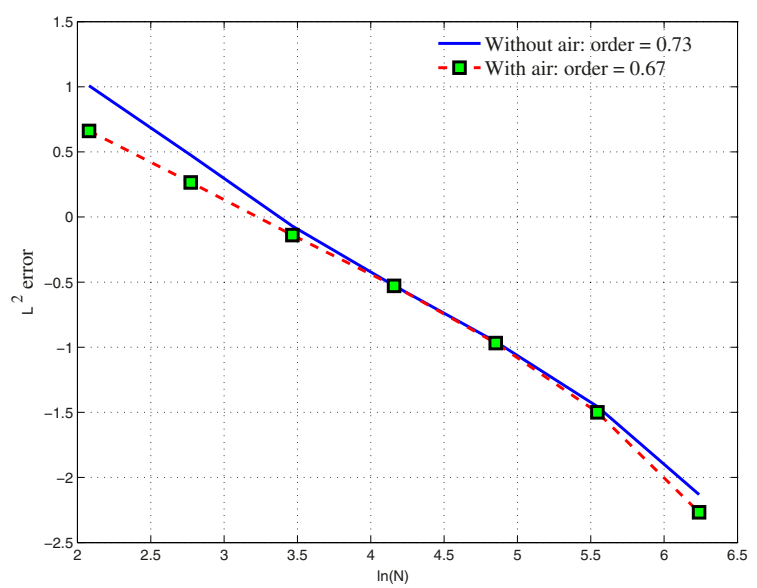

(c) Numerical order

FiguRE 13. The "dam-break" problem at $t=0.1$ s. $L^{2}$ norm of the error (in log scale). 


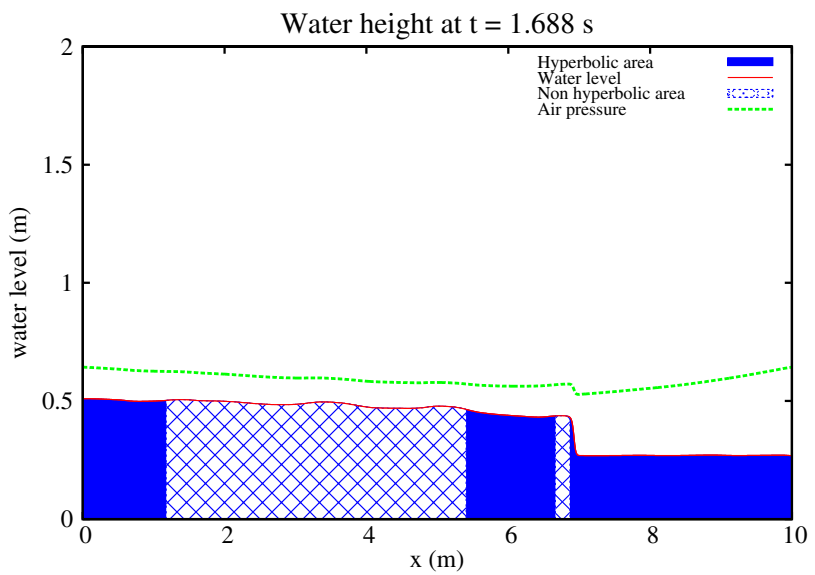

(a) Two-layer model

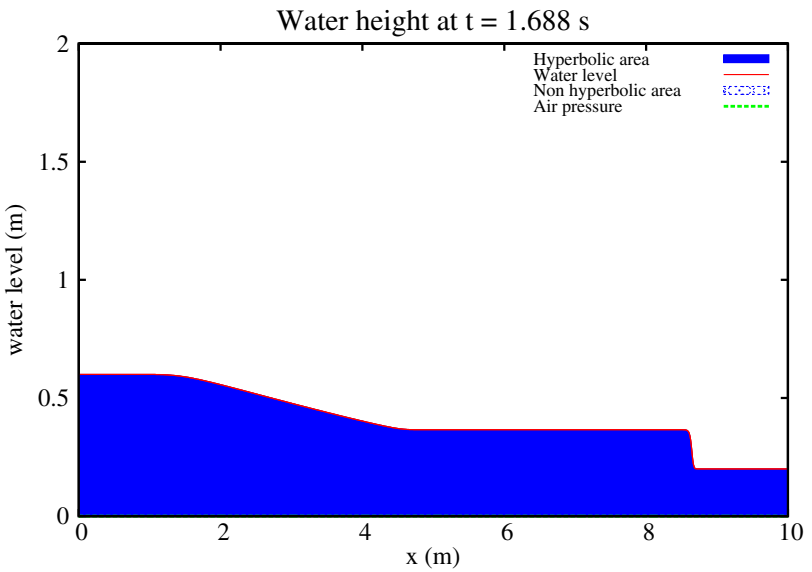

(b) The single fluid

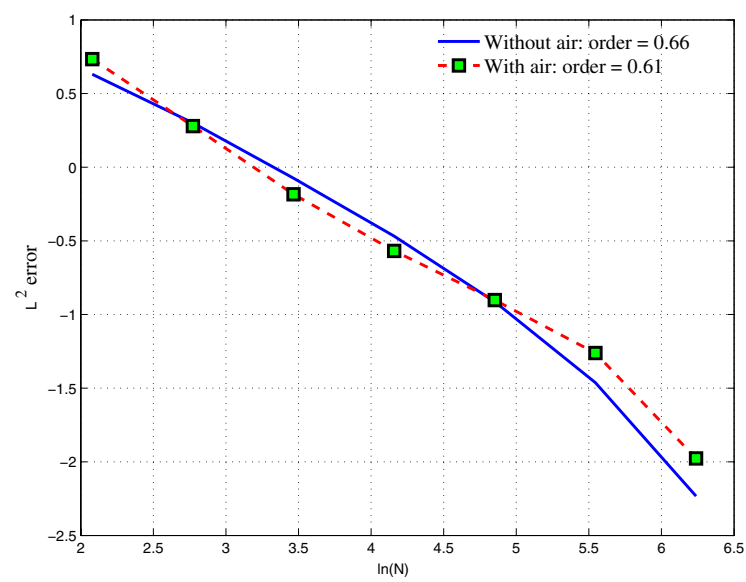

(c) Numerical order

Figure 14. The "dam-break" problem at $t=1.688 \mathrm{~s} . L^{2}$ norm of the error (in log scale). 
system is hyperbolic, Figure 13 displays the case where the system is non hyperbolic and finally in Figure 14, the system is partially hyperbolic.

Even if the system loses its hyperbolicity, the numerical order of the two-layer kinetic scheme is very close to the case of "single fluid" for the $L^{2}$-norm as well as for the $L_{t}^{1}\left(L_{x}^{2}\right)$ (see Fig. 11b) which provides a numerical stability as the space step goes to 0 .

Finally, even if we do not know if the presented numerical scheme preserves the entropy inequalities, this stability should come from the fact that the system at continuous level preserves an entropy equality (see Thm. 2.3).

\section{Conclusion}

This study is of course a first step in the comprehension and the modelisation of the air role in the transient flows in closed pipes. The kinetic scheme seems to be well-adpated to treat a two layer model even if the obtained partial differential system is conditionally hyperbolic.

We have now to treat the air entrapment pocket which may be encountered in a rapid filling process of a closed pipe: in this case a portion of the pipe will be completely filled and an air pocket will be entrapped. This is the next step of our research work, since in previous works we have derived a model for pressurised flow or mixed flows in closed pipes without taking into account the role of the air and proposed a Roe like Finite Volume method and a kinetic scheme [6-9,11].

Next, we have to deal with the evaporation/condensation of gas or water when it is not supposed to be isothermal and later, because in the water hammer phenomenon, large depression may occur, we have to deal with the natural cavitation problem.

Acknowledgements. This work is supported by the "Agence Nationale de la Recherche" referenced by ANR-08-BLAN0301-01 and the second author was supported by the ERC Advanced Grant FP7-246775 NUMERIWAVES. This work was finalized while the third author was visiting BCAM-Basque Center for Applied Mathematics, Derio, Spain, and partially supported by the ERC Advanced Grant FP7-246775 NUMERIWAVES. The third author wishes to thank Enrique Zuazua for his kind hospitality. The authors thank the referees for their valuable remarks which led to substantial improvement of the first version of this paper.

\section{REFERENCES}

[1] R. Abgrall and S. Karni, Two-layer shallow water system: a relaxation approach. SIAM J. Sci. Comput. 31 (2009) $1603-1627$.

[2] E. Audusse, A multilayer Saint-Venant model: derivation and numerical validation. Discrete Contin. Dyn. Syst. Ser. B $\mathbf{5}$ (2005) 189-214.

[3] R. Barros and W. Choi, On the hyperbolicity of two-layer flows, in Frontiers of applied and computational mathematics. World Sci. Publ., Hackensack, NJ (2008) 95-103.

[4] F. Bouchut, Nonlinear stability of finite volume methods for hyperbolic conservation laws and well-balanced schemes for sources, in Frontiers in Mathematics. Birkhäuser Verlag, Basel (2004)

[5] F. Bouchut and T. Morales, An entropy satisfying scheme for two-layer shallow water equations with uncoupled treatment. ESAIM: M2AN 42 (2008) 683-689.

[6] C. Bourdarias and S. Gerbi, A finite volume scheme for a model coupling free surface and pressurised flows in pipes. J. Comput. Appl. Math. 209 (2007) 109-131.

[7] C. Bourdarias, M. Ersoy and S. Gerbi, A kinetic scheme for pressurised flows in non uniform closed water pipes. Monografias de la Real Academia de Ciencias de Zaragoza 31 (2009) 1-20.

[8] C. Bourdarias, M. Ersoy and S. Gerbi, A model for unsteady mixed flows in non uniform closed water pipes and a well-balanced finite volume scheme. International Journal on Finite Volumes 6 (2009) 1-47.

[9] C. Bourdarias, M. Ersoy and S. Gerbi, A kinetic scheme for transient mixed flows in non uniform closed pipes: a global manner to upwind all the source terms. J. Sci. Comput. (2011) 1-16.

[10] C. Bourdarias, M. Ersoy and S. Gerbi, A mathematical model for unsteady mixed flows in closed water pipes. Science China Math. 55 (2012) 221-244.

[11] C. Bourdarias, M. Ersoy and S. Gerbi, Unsteady mixed flows in non uniform closed water pipes: a Full kinetic Approach (2011). Submitted.

[12] M. Castro, J. Macías and C. Parés, A $Q$-scheme for a class of systems of coupled conservation laws with source term. Application to a two-layer 1-D shallow water system. ESAIM: M2AN 35 (2001) 107-127. 
[13] S. Cerne, S. Petelin and I. Tiselj, Coupling of the interface tracking and the two-fluid models for the simulation of incompressible two-phase flow. J. Comput. Phys. 171 (2001) 776-804.

[14] M.H. Chaudhry, S.M. Bhallamudi, C.S. Martin M. and Naghash, Analysis of transient pressures in bubbly, homogeneous, gas-liquid mixtures. J. Fluids Eng. 112 (1990) 225-231.

[15] C.M. Dafermos, Generalized characteristics in hyperbolic systems of conservation laws. Arch. Ration. Mech. Anal. 107 (1989) $127-155$.

[16] G. Dal Maso, P.G. Lefloch and F. Murat, Definition and weak stability of nonconservative products. J. Math. Pures Appl. 74 (1995) 483-548.

[17] M. Ersoy, Modélisation, analyse mathématique et numérique de divers écoulements compressibles ou incompressibles en couche mince. Ph.D. thesis, Université de Savoie, Chambéry (2010).

[18] I. Faille and E. Heintze, A rough finite volume scheme for modeling two-phase flow in a pipeline. Comput. Fluids 28 (1999) $213-241$.

[19] A.T. Fuller, Root location criteria for quartic equations. IEEE Trans. Autom. Control 26 (1981) 777-782.

[20] J.-F. Gerbeau and B. Perthame, Derivation of viscous Saint-Venant system for laminar shallow water; numerical validation. Discrete Contin. Dyn. Syst. Ser. B 1 (2001) 89-102.

[21] M.A. Hamam and A. McCorquodale, Transient conditions in the transition from gravity to surcharged sewer flow. Can. J. Civ. Eng. 9 (1982) 189-196.

[22] T. Hibiki and M. Ishii, One-dimensional drift-flux model and constitutive equations for relative motion between phases in various two-phase flow regimesaa. Int. J. Heat Mass Transfer 46 (2003) 4935-4948.

[23] T. Hibiki and M. Ishii, Thermo-fluid dynamics of two-phase flow. With a foreword by Lefteri H. Tsoukalas. Springer, New York (2006).

[24] L.V. Ovsjannikov, Models of two-layered "shallow water". Zh. Prikl. Mekh. i Tekhn. Fiz. 180 (1979) 3-14.

[25] C. Parés, Numerical methods for nonconservative hyperbolic systems: a theoretical framework. SIAM J. Numer. Anal. 44 (2006) 300-321.

[26] B. Perthame and C. Simeoni, A kinetic scheme for the Saint-Venant system with a source term. Calcolo 38 (2001) $201-231$.

[27] L. Sainsaulieu, An Euler system modeling vaporizing sprays, in Dynamics of Hetergeneous Combustion and Reacting Systems, Progress in Astronautics and Aeronautics, AIAA, Washington, DC 152 (1993).

[28] L. Sainsaulieu, Finite volume approximate of two-phase fluid flows based on an approximate Roe-type Riemann solver. J. Comput. Phys. 121 (1995) 1-28.

[29] S.B. Savage and K. Hutter, The motion of a finite mass of granular material down a rough incline. J. Fluid Mech. 199 (1989) $177-215$

[30] C. Savary, Transcritical transient flow over mobile beds, boundary conditions treatment in a two-layer shallow water model. Ph.D. thesis, Louvain (2007).

[31] J.B. Schijf and J.C. Schönfled, Theoretical considerations on the motion of salt and fresh water, in Proc. of Minnesota International Hydraulic Convention. IAHR (1953) 322-333.

[32] C.S.S. Song, Two-phase flow hydraulic transient model for storm sewer systems, in Second international conference on pressure surges, BHRA Fluid engineering. Bedford, England (1976) 17-34.

[33] C.S.S. Song, Interfacial boundary condition in transient flows, in Proc. of Eng. Mech. Div. ASCE, on advances in civil engineering through engineering mechanics (1977) 532-534.

[34] C.S.S. Song, J.A. Cardle and K.S. Leung, Transient mixed-flow models for storm sewers. J. Hydraul. Eng. 109 (1983) 14871503.

[35] H.B. Stewart and B. Wendroff, Two-phase flow: models and methods. J. Comput. Phys. 56 (1984) $363-409$.

[36] I. Tiselj and S. Petelin, Modelling of two-phase flow with second-order accurate scheme. J. Comput. Phys. 136 (1997) 503-521.

[37] D.C. Wiggert and M.J. Sundquist, The effects of gaseous cavitation on fluid transients. J. Fluids Eng. 101 (1979) $79-86$.

[38] E.B. Wylie and V.L. Streeter, Fluid transients in systems. Prentice Hall, Englewood Cliffs, NJ (1993). 